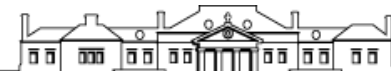

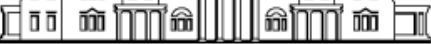

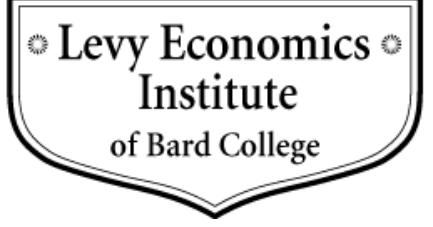

Working Paper No. 944

\title{
The Empirics of Canadian Government Securities Yields
}

\author{
by
}

\author{
Tanweer Akram* \\ Thrivent
}

and

Anupam Das

Mount Royal University

January 2020

\begin{abstract}
Acknowledgements: The authors thank their colleagues for comments and suggestions. They also thank Ms. Mary Rafferty and Ms. Elizabeth Dunn for their assistance in copy editing. Disclaimer: The authors' institutional affiliations are provided solely for identification purposes. Views expressed are solely those of the authors and are not necessarily those of Thrivent, Thrivent Asset Management, or any affiliates. This is for information purposes only and should not be construed as an offer to buy or sell any investment product or service. Disclosure: Tanweer Akram's employer, Thrivent, invests in a wide range of securities. Asset management services are provided by Thrivent Asset Management, LLC, a wholly owned subsidiary of Thrivent, the marketing name for Thrivent Financial for Lutherans, Appleton, Wisconsin. Securities and investment advisory services are offered through Thrivent Investment Management Inc., a wholly owned subsidiary of Thrivent. For additional important information, visit Thrivent.com/disclosures.

Dataset availability: The dataset used in the empirical part of this paper is available upon request to bona fide researchers for the replication and verification of the results.
\end{abstract}

The Levy Economics Institute Working Paper Collection presents research in progress by Levy Institute scholars and conference participants. The purpose of the series is to disseminate ideas to and elicit comments from academics and professionals.

Levy Economics Institute of Bard College, founded in 1986, is a nonprofit, nonpartisan, independently funded research organization devoted to public service. Through scholarship and economic research it generates viable, effective public policy responses to important economic problems that profoundly affect the quality of life in the United States and abroad.

Levy Economics Institute P.O. Box 5000

Annandale-on-Hudson, NY 12504-5000

http://www.levyinstitute.org

Copyright (C Levy Economics Institute 2020 All rights reserved

ISSN 1547-366X 


\begin{abstract}
Keynes argued that the short-term interest rate is the main driver of the long-term interest rate. This paper empirically models the relationship between short-term interest rates and long-term government securities yields in Canada, after controlling for other important financial variables. The statistical analysis uses high-frequency daily data from 1990 to 2018. It applies both the cointegration technique and Granger causality within the vector error correction (VEC) framework. The empirical results suggest that the action of the monetary authority is an important determinant of Canadian government securities yields, which supports the Keynesian perspective. These findings have important implications for investors, financial analysts, and policymakers.
\end{abstract}

KEYWORDS: Canadian Government Bond Yields; Long-Term Interest Rate; Short-Term Interest Rate; Monetary Policy; Cointegration; Granger Causality

JEL CLASSIFICATIONS: E43; E50; E60; G10; G12 


\section{INTRODUCTION}

\section{Motivation}

The long-term interest rate on Canadian government securities is an important theoretical and empirical topic. It is germane for macroeconomic theory and public policy, particularly as it concerns these issues: the monetary transmission mechanism, monetary policy, market volatility, inflationary pressures, financial conditions, government debt management and operations, and the effects of higher government debt and deficits ratios on government securities yields. Understanding the empirics of Canadian government securities yields can be useful for investors and portfolio managers in making strategic and tactical asset allocation and investment decisions concerning duration, convexity, speculation, and delta hedging.

John Maynard Keynes (1930, 352-364) argued that the central bank's actions have a decisive influence on the long-term interest rate. He claimed that the central bank's policy rate sets the short-term interest rate, which has a crucial influence on the long-term interest rate for government securities. Keynes wrote $(1930,353)$ : "[T]he influence of the short-term rate of

interest on the long-term rate is much greater than anyone ... would have expected." $\mathrm{He}$ attributed this correlation to fundamental macroeconomic factors, technical characteristics of financial markets, and investors' behavior, including herding and the formation of expectations. Keynes $(1930,363)$ asserted that "there is no reason to doubt the ability of a Central Bank to make its short-term rate of interest effective in the market."

This paper empirically examines whether Keynes's claim holds true. It analyzes the effects of the short-term interest rate on the long-term interest rate, as measured by Canadian government securities yields. The statistical exercise undertaken in this paper controls for several important factors, including the domestic equity market, oil prices, and the exchange rate of the Canadian dollar. This paper uses daily data to analyze Canadian government securities yields. There are two main benefits of using daily financial data. First, daily data over a long period provides many observations, which ensures a robust degree of freedom. Second, analyzing high-frequency data provides a near real-time fundamental assessment of long-term government securities 
yields, and thus provides important information to investors, financial analysts, and policymakers.

\section{Relation to Debates in the Literature}

This paper contributes to the ongoing debate on the dynamics of government bond yields. The literature on government bond yields contains many substantial but unresolved debates. The two main schools of thought regarding the dynamics of government bond yields represent the neoclassical and Keynesian views.

The neoclassical view holds that government bond yields are the outcome of the demand for and supply of loanable funds. Other exogenous factors, such as government debt and deficit ratios, also influence government bond yields. In the past two decades, scholars have presented their arguments in various studies on the dynamics of government bond yields and various macroeconomic and financial variables, including government debt and deficit ratios. There are numerous studies from the neoclassical perspective. For examples, see Ardagna, Caselli, and Lane (2007); Baldacci and Kumar (2010); Doi, Hoshi, and Okimoto (2011); Elmendort and Mankiw (1998); Gurber and Kamin (2012); Hansen and İmrohoroğlu (2013); Horioka, Nomoto, and Tera-Hagiwara (2014); Hoshi and Ito (2013, 2014); Lam and Tokuoka (2011); Tokuoka (2012); Paccagnini (2016); Poghosyan (2014); Reinhart and Rogoff (2019); and Tkačevs and Vilerts (2019). Their analyses and interpretation of the data support the neoclassical view.

The Keynesian school follows Keynes's ([1936] 2007) argument that interest rates have a psychological and sociological foundation in a world characterized by ontological uncertainty (Davidson 2015). The Keynesian school maintains the liquidity preference view of interest rates as articulated in Keynes's General Theory ([1936] 2007). This view holds that the long-term interest rate is primarily determined by the central bank's actions, such as the setting of benchmark policy rates, repurchase and reverse repurchase agreements, forward guidance about policy rates, and decisions concerning the central bank's monetary base and balance sheets. Riefler's (1930) analysis of the dynamics of the short-term interest rate and the long-term interest rate in the United States in the 1920s and 1930s provided the empirical basis for Keynes to formulate this hypothesis. The Keynesian perspective on interest rates and monetary operations, 
and their relation to fiscal policy, was later developed in Lerner (1947). In recent years, several Keynesian and Post-Keynesian economists have advanced the Keynesian view of interest rates. They argued that an increase (decrease) in government debt and deficit ratios does not necessarily lead to higher (lower) government bond yields, particularly in countries with monetary sovereignty (Akram 2014; Kregel 2011; Lavoie 2014; Wray 2012). This is contrary to the claims of the neoclassical view. The strong relationship between the short-term interest rate and the long-term interest rate on government bonds has been empirically shown to hold in several studies. Akram and Das (2014) and Akram and Li (2018, 2019a) argued this for Japan; Akram and Das (2015, 2019a) for India; Akram and Das (2017) for the eurozone countries; Akram and Li (2016, 2017, 2019b), Akram and Das (2019b), and Levrero and Deleidi (2019) for the United States; Akram and Das (forthcoming) for Australia; and Simoski (2019) for several Latin American countries, including Brazil and Mexico.

The aim of this paper is to empirically examine the Canadian case from a Keynesian perspective. This paper contributes to the debate in the empirical literature on government securities yields by analyzing the relationship between short-term interest rates, government securities yields, and other macroeconomic and financial variables in Canada. High-frequency daily data are used here for analyzing the relationship between the short-term interest rate and the long-term interest rate on Canadian government bonds. Very few papers use such high-frequency daily data to study government securities yields (Bollerslev, Cai, and Song 2000; Gürkaynak, Sack, and Wright 2007). Using the higher frequency data to examine the empirics of Canadian government bond yields from a Keynesian perspective is a useful extension of the literature because it furthers the ongoing debate. This is the first paper to use daily data in analyzing Canadian government securities yields from a Keynesian perspective. The findings of this paper are well-aligned with the Keynesian perspective because the results suggest that the short-term interest rate is the key determinant of the long-term interest rate on Canadian government securities.

\section{Outline of the Paper}

This paper proceeds as follows. Section 2 provides an overview of the evolution of Canadian government securities yields and key financial variables. Section 3 presents the data used in the paper. It also undertakes unit root tests, describes the econometric methodology, and reports the 
empirical findings. Section 4 concludes with a summary of the findings and their relevance to debates in economic theory and policy.

\section{THE EVOLUTION OF CANADIAN GOVERNMENT SECURITIES YIELDS AND KEY FINANCIAL VARIABLES}

It is useful to look at the evolution of Canadian government securities yields and key macroeconomic variables in order to understand the underlying dynamics between these variables. It can also give a useful perspective about the drivers of the long-term interest rate and the underlying relationships between these macroeconomic and financial variables.

Figure 1 shows the evolution of long-term Canadian government securities yields. It shows that government bond yields have progressively declined over time. There appears to be an underlying trend. The decline in government securities yields is partly due to a decline in observed inflation and inflation expectations. Government securities yields were elevated in the early 1990s. Since peaking around 12 percent in the early 1990s, yields have steadily declined. Yields rose sharply in the mid-1990s. Since then, bond yields have steadily declined, though there have been modest increases from time to time. Government securities yields fell notably prior to the global financial crisis. An attentive look at government securities yields' evolution and relating this to concurrent macroeconomic events and trends can be useful in understanding the underlying dynamics of the government securities market. 
Figure 1: The Evolution of Yields of Selected Long-Term Canadian Government Securities, 1990-2018

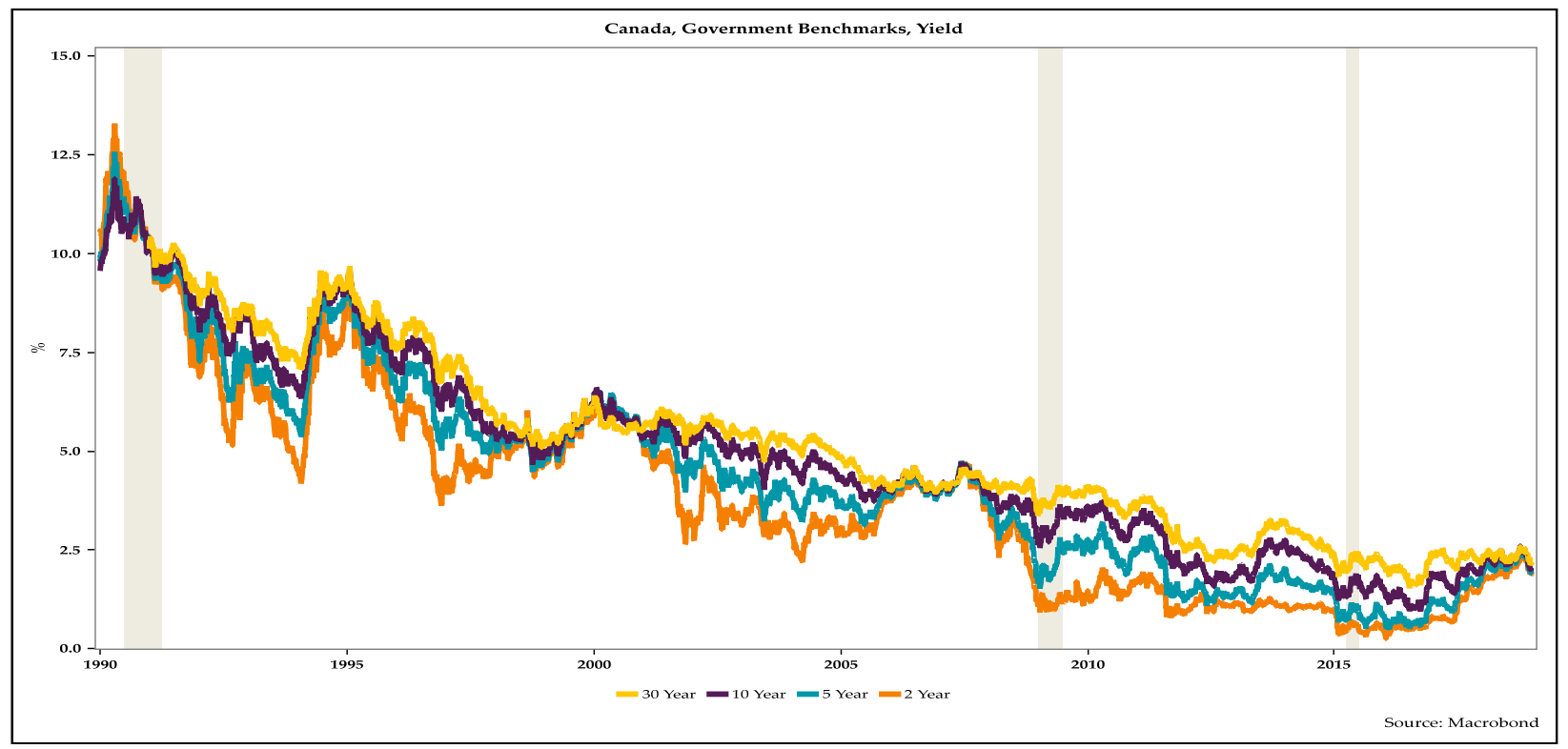

Figure 2 displays the evolution of the central bank's policy rate and the short-term interest rate, as measured by the yield of 3-month Treasury bills. It shows that the yield of 3-month Treasury bills generally moves in tandem with the Bank of Canada's policy rate.

Figure 2: The Evolution of the Policy Rate and the Short-Term Interest Rate in Canada, 1990-2018

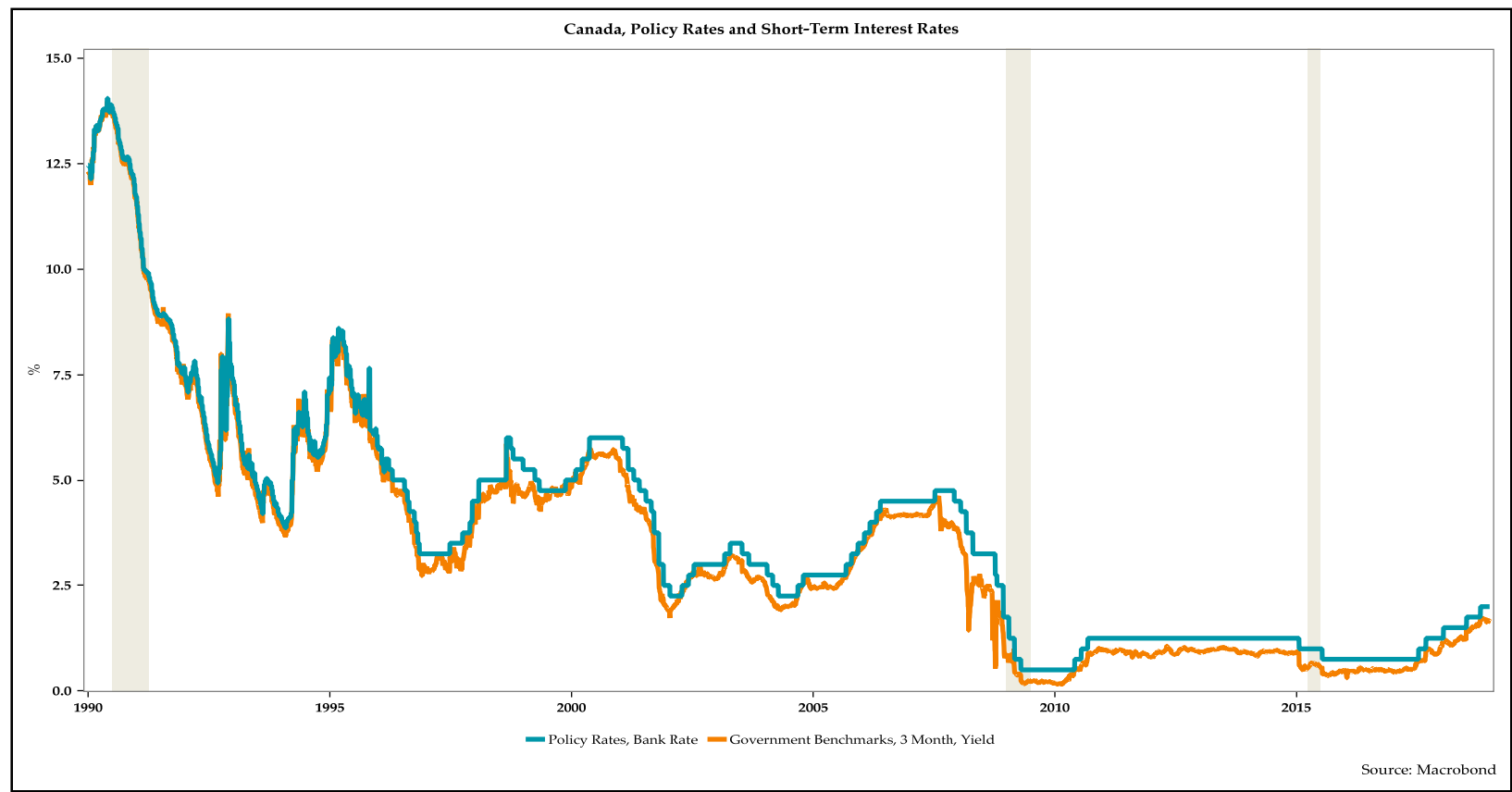


Figure 3 shows the evolution of Canadian equity prices, as measured by Standard and Poor's (S\&P) Index for the Toronto Stock Exchange (TSX).

Figure 3: The Evolution of the Canadian Equity Price Index, 1990-2018

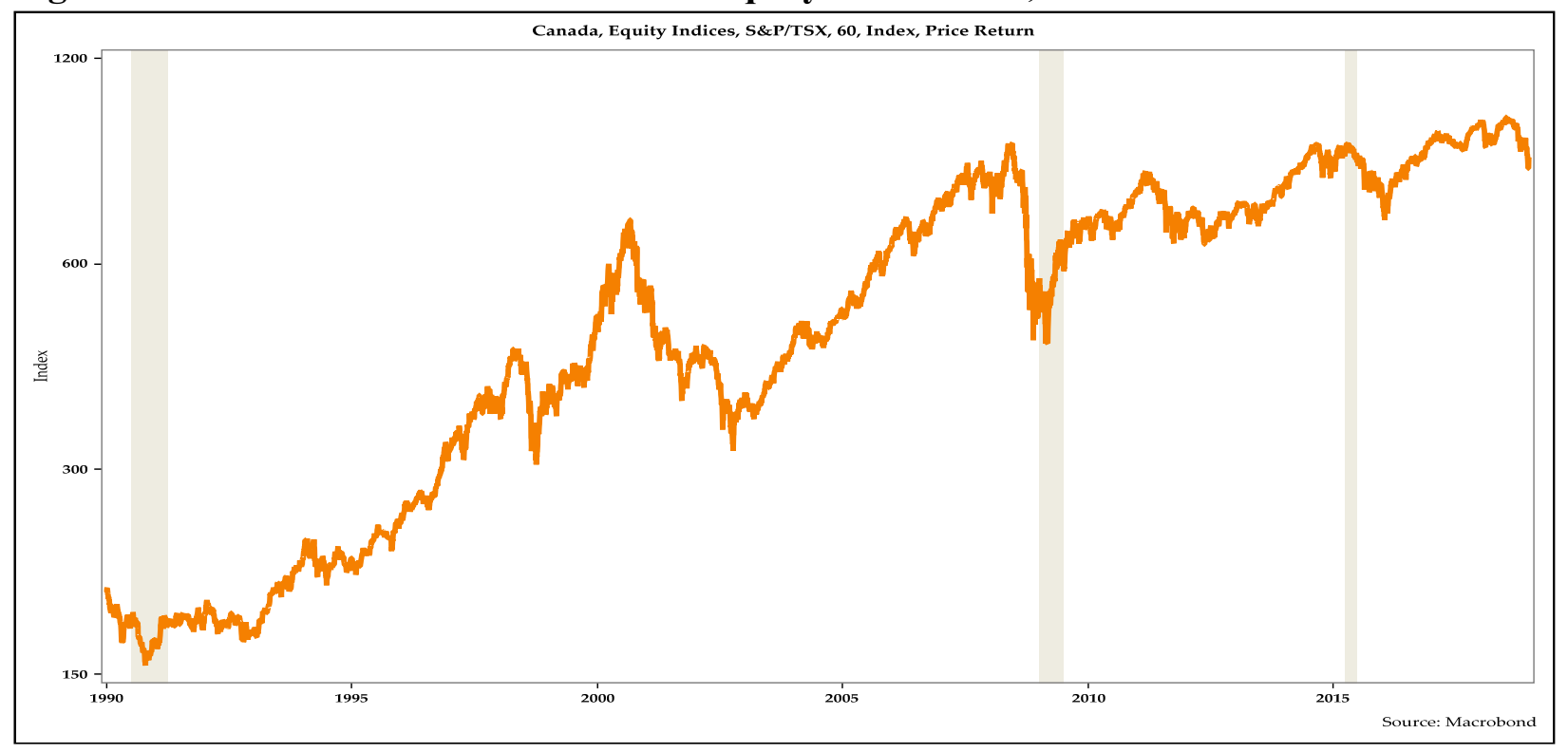

Figure 4 exhibits the evolution of crude oil prices. Monitoring this evolution is useful because oil prices provide information about inflationary pressures emanating from energy inputs. Crude oil prices also provide insights about growth in the global economy and the outlook for global effective demand. Finally, oil prices are important indicators of economic and political risks, particularly related to conditions in the major crude oil producing areas. Higher crude oil prices should increase government securities yields if investors regard higher crude oil prices as a harbinger of domestic and global inflationary pressures and/or strong effective demand in the global economy. 
Figure 4: The Evolution of Crude Oil Prices, 1990-2018

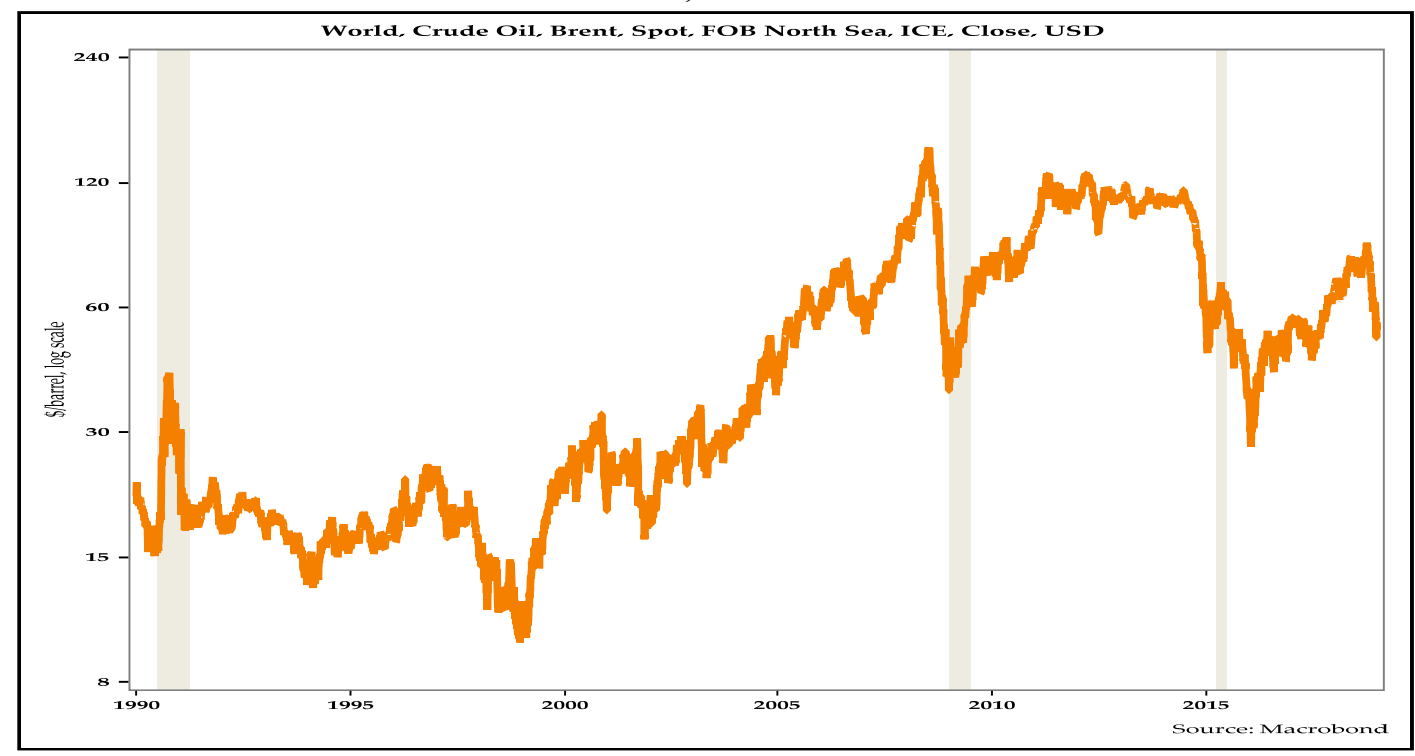

Figure 5 shows the evolution of the value of the Canadian dollar. The currency value can provide clues to investor confidence and sentiments, as well as to financial flows - all of which have a bearing on Canadian government securities yields. The USD/CAD exchange rate is expressed in terms of Canadian dollar per US dollar. This means that a rise in the exchange rate is a depreciation of the Canadian dollar with respect to the US dollar, while a decline in the exchange rate is an appreciation of the Canadian dollar with respect to the US dollar.

Figure 5: The Evolution of the USD/CAD Exchange Rate, 1990-2018

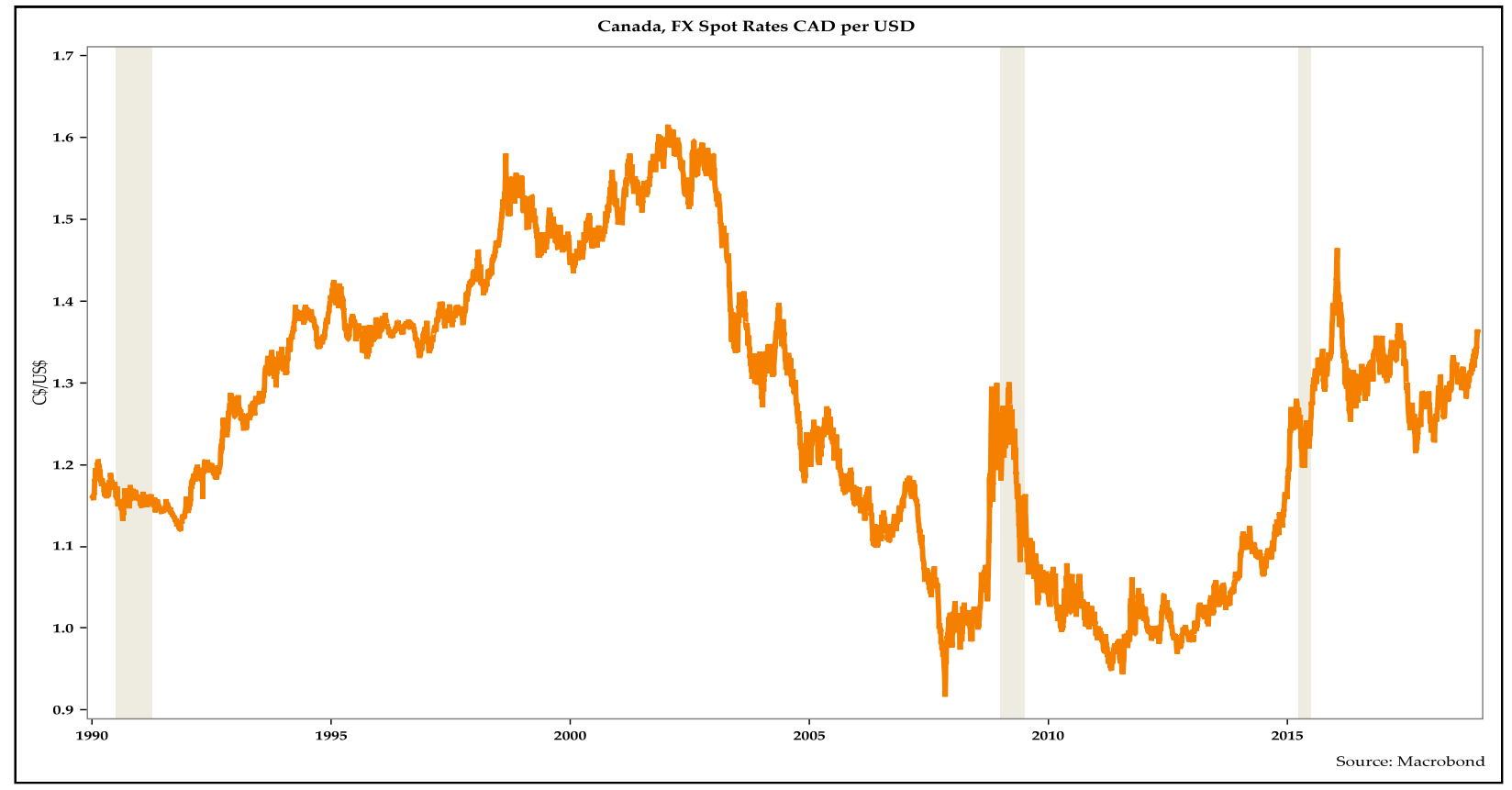


Figures 6 to 11 are scatterplots. They show the correlations between the yields of Canadian government securities of various maturity tenors and the yields of 3-month Treasury bills. These scatterplots reveal two clear and distinct patterns. First, there is a strong positive correlation between the yields of long-term Canadian government securities and Treasury bills. Second, the strong positive correlation between the yields of long-term Canadian government securities and Treasury bills declines as the maturity tenor of Canadian government securities increases.

Figure 6: Scatterplot of the Yields of 2-Year Canadian Government Securities and 3Month Treasury Bills

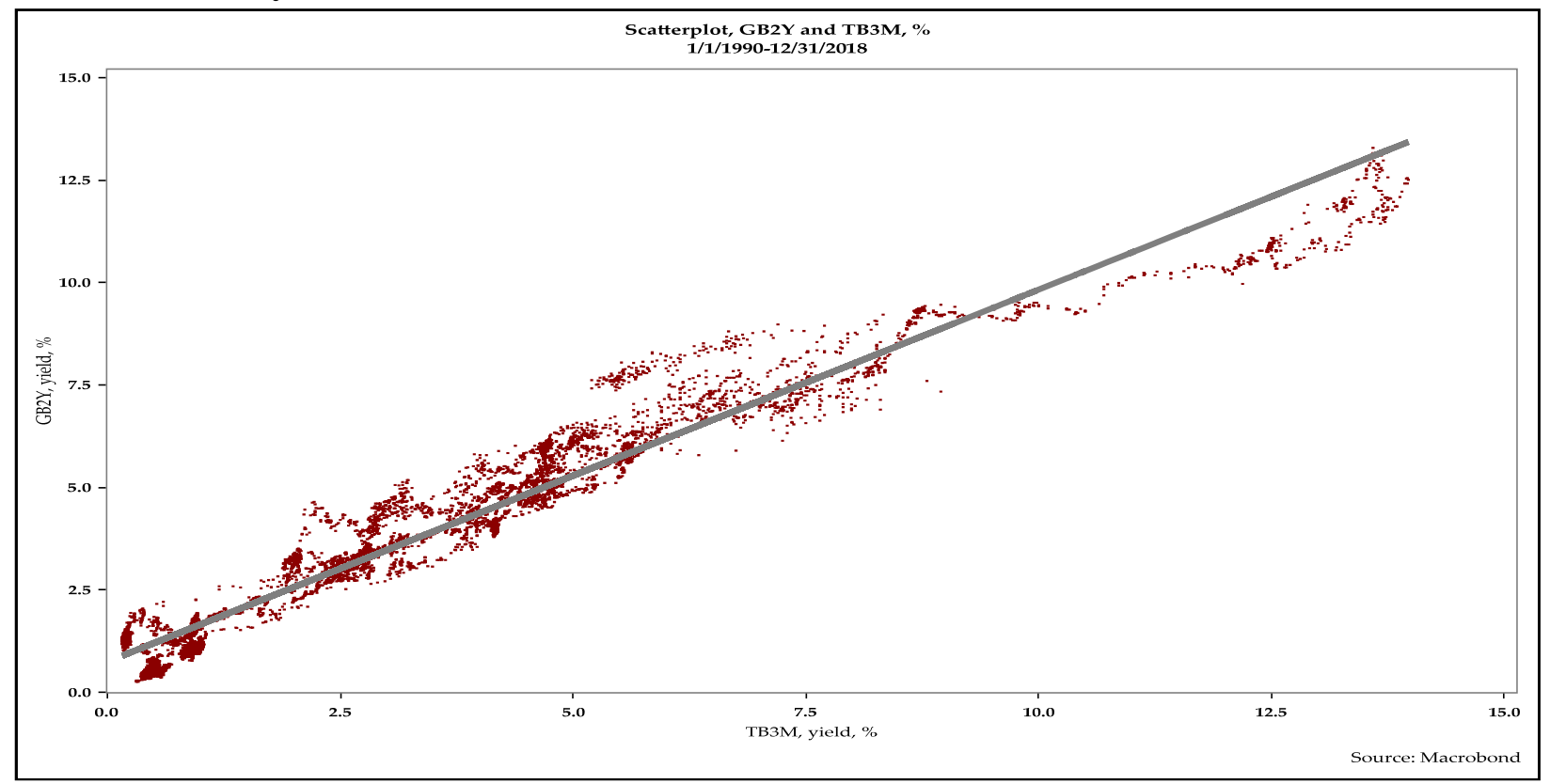


Figure 7: Scatterplot of the Yields of 3-Year Canadian Government Securities and 3Month Treasury Bills

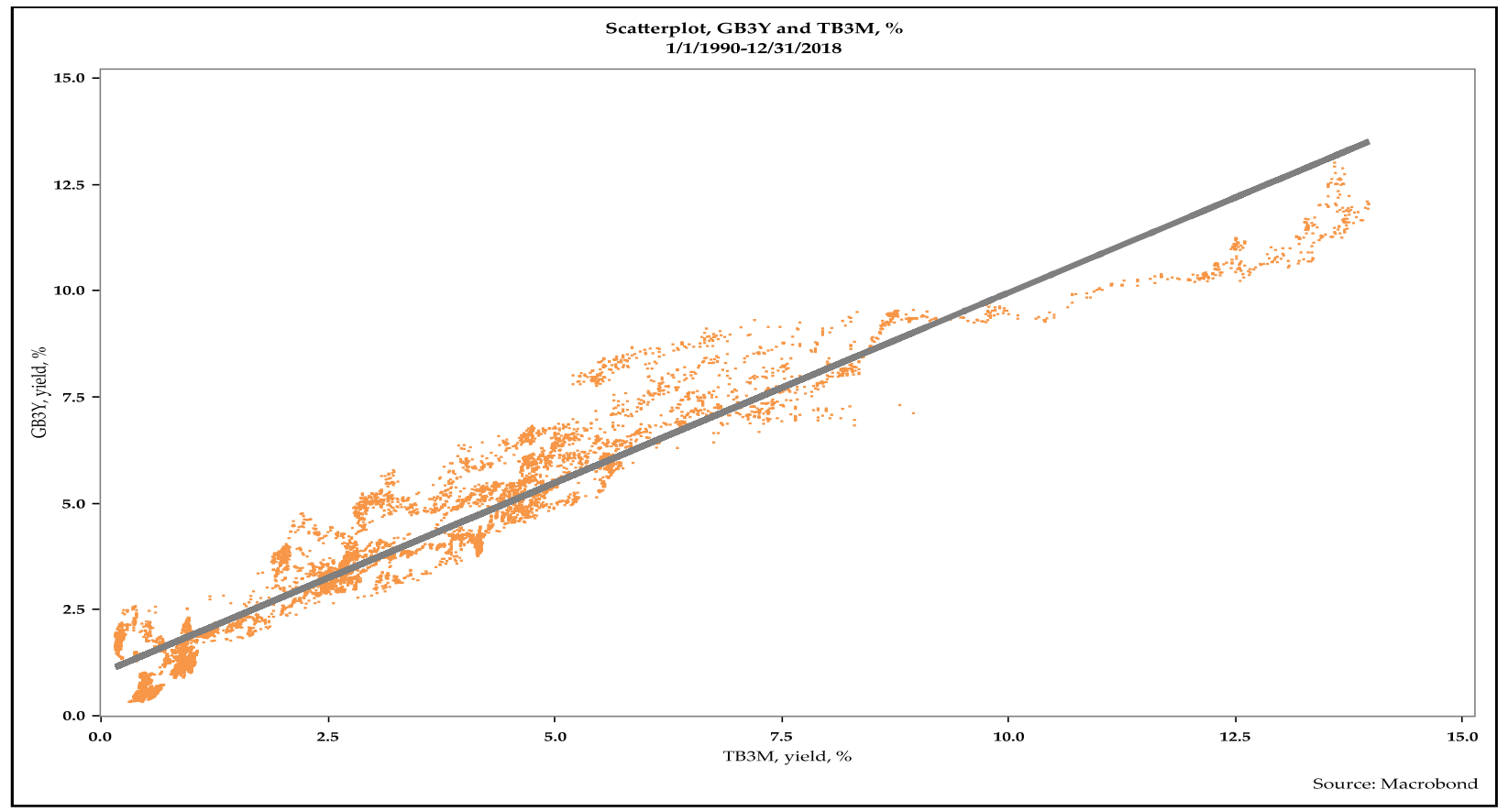

Figure 8: Scatterplot of the Yields of 5-Year Canadian Government Securities and 3Month Treasury Bills

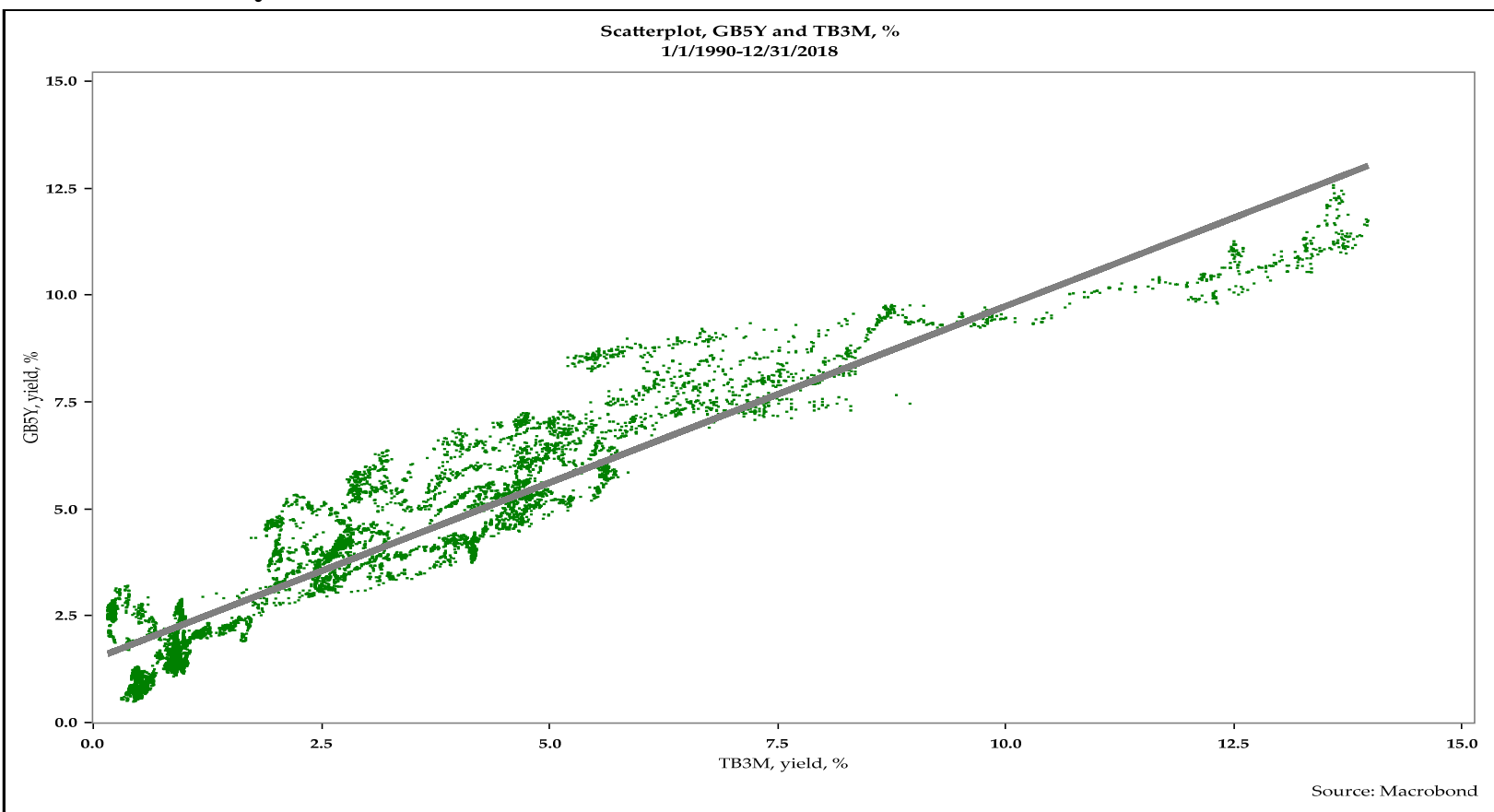


Figure 9: Scatterplot of the Yields of 7-Year Canadian Government Securities and 3Month Treasury Bills

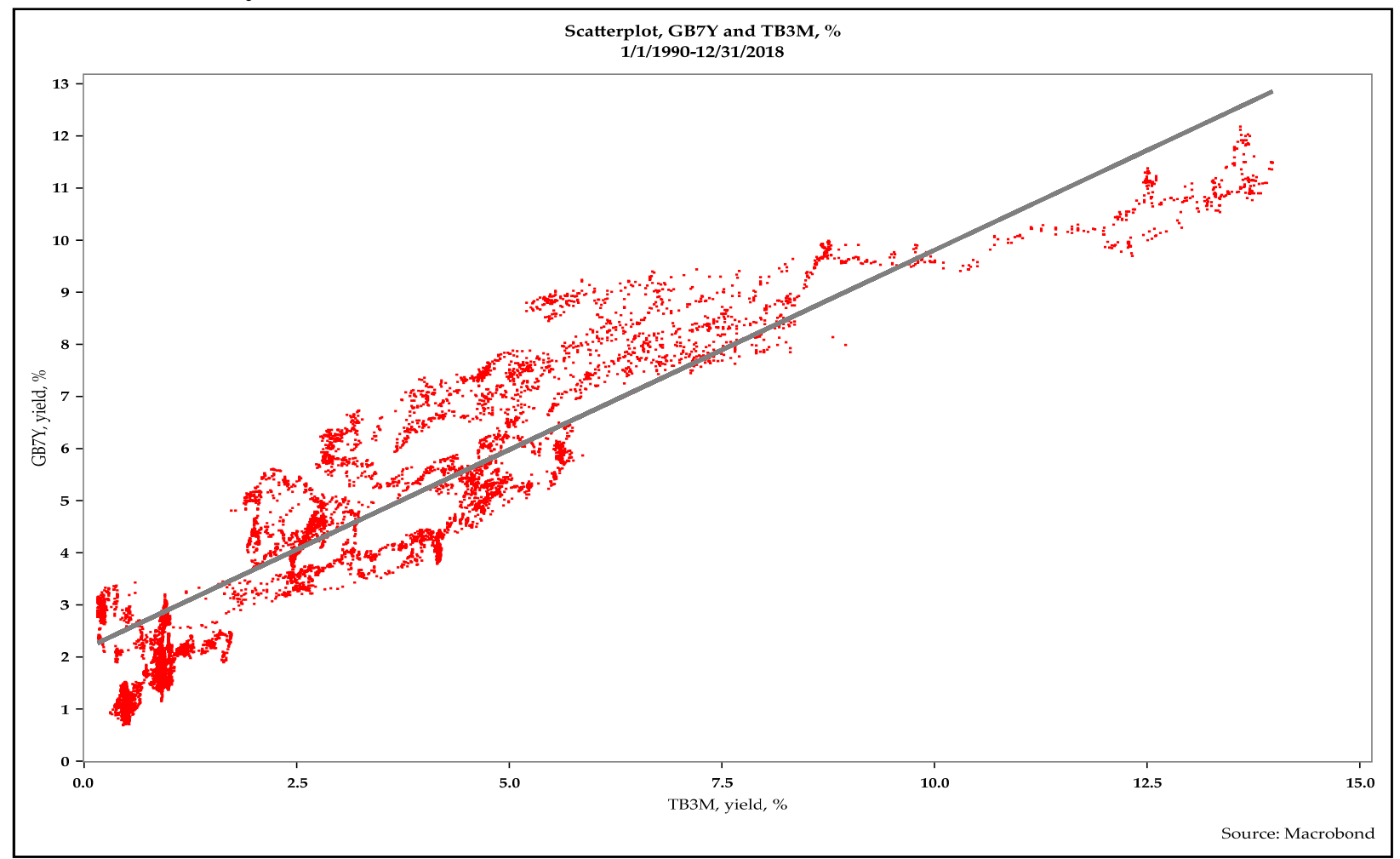

Figure 10: Scatterplot of the Yields of 10-Year Canadian Government Securities and 3Month Treasury Bills

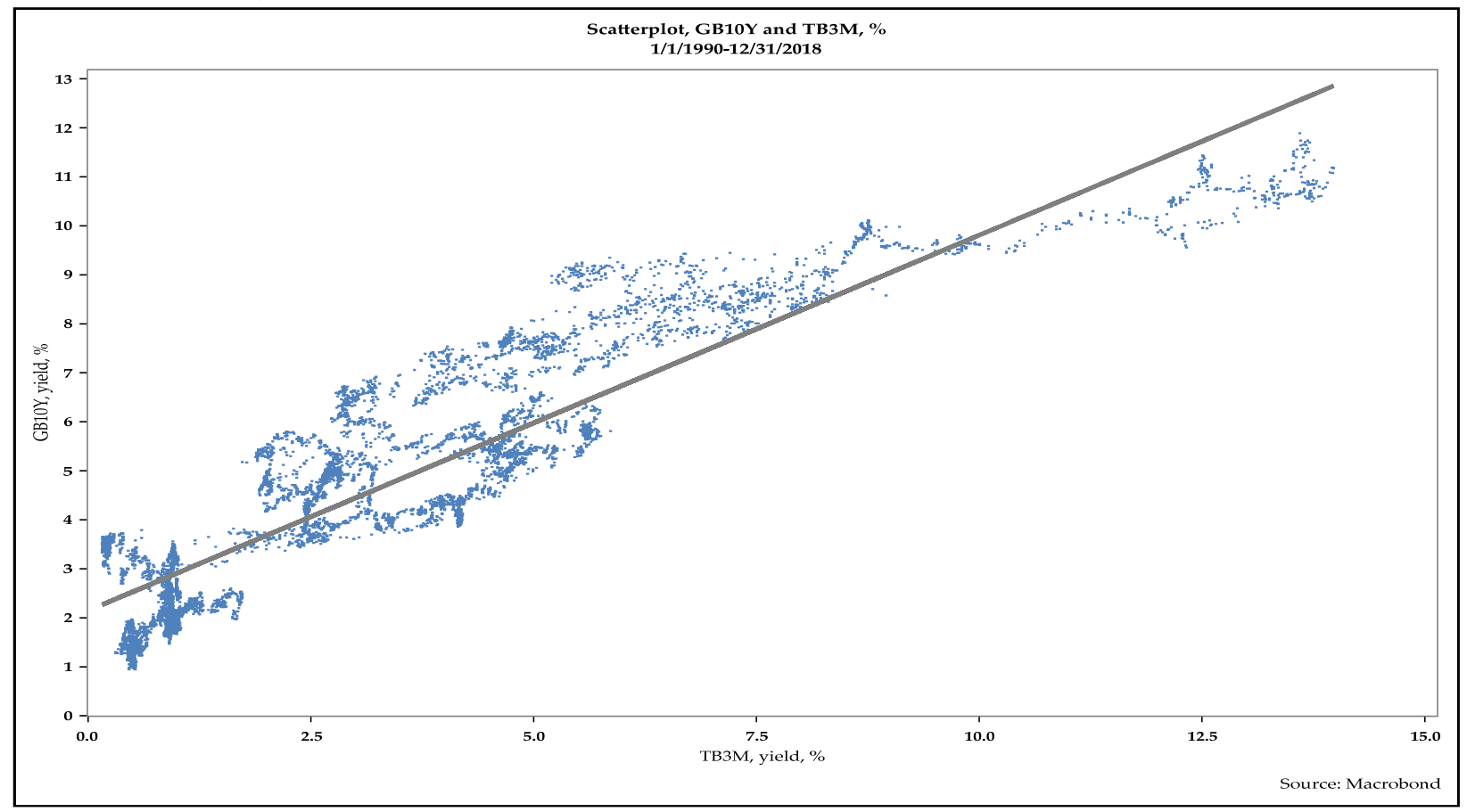


Figure 11: Scatterplot of the Yields of 30-Year Canadian Government Securities and 3Month Treasury Bills

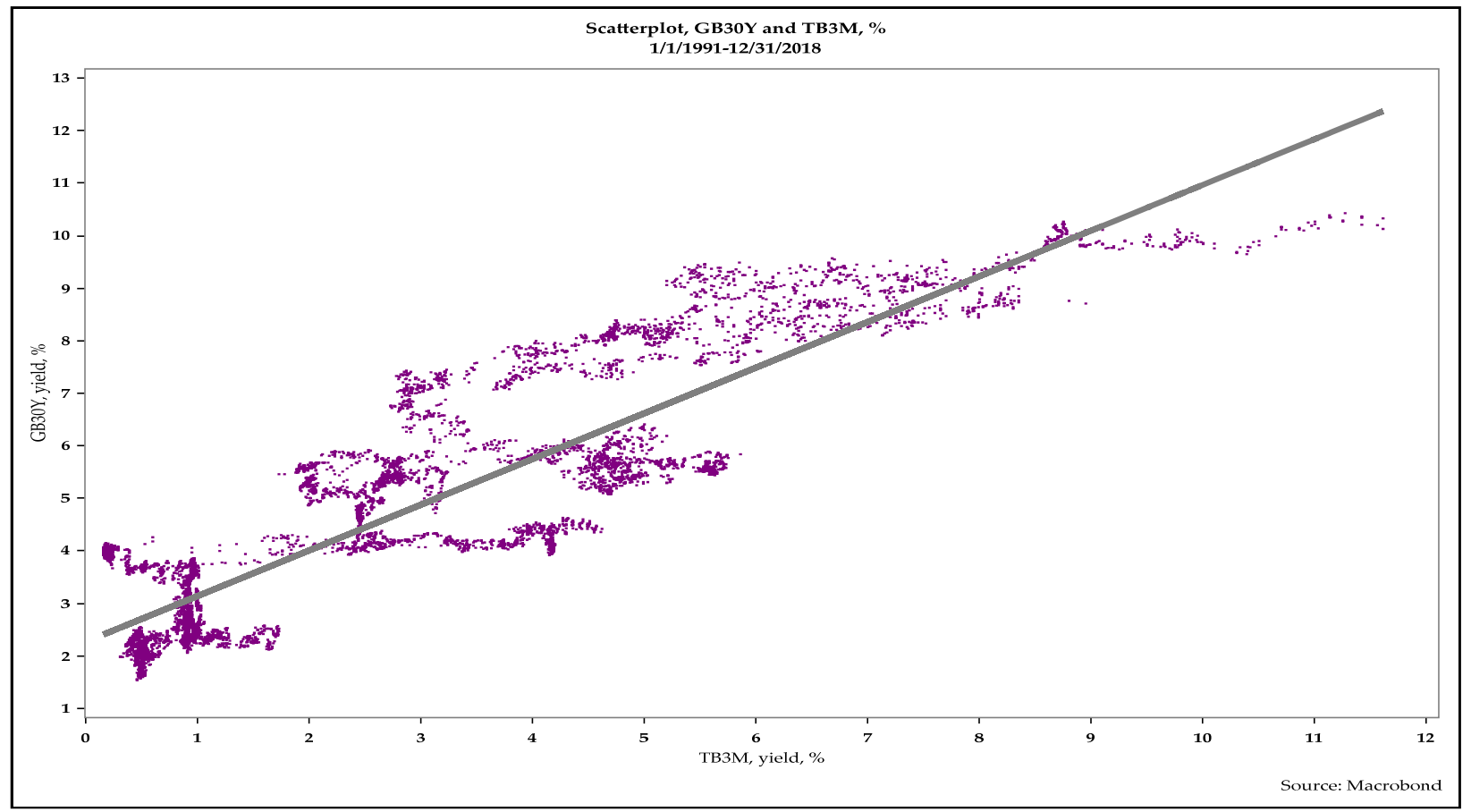

\section{DATA, METHODS, AND EMPIRICAL FINDINGS}

\section{Data and Methods}

Table 1, below, summarizes the data used in this paper. The first column lists the variables and their names. The second column describes the data and provides the data's date range. The third column displays the frequency of the data. The final column lists the data sources. The dataset begins on January 1, 1990 and ends on December 31, 2018.

Since the variables in the dataset are defined over a long period of time, it is important to identify whether these variables are stationary. If they are found to be nonstationary, applying the standard least squares technique would be an inappropriate approach. The order of variable integration is tested using both augmented Dickey-Fuller (ADF) (Dickey and Fuller 1979, 1981) and Phillips-Perron (PP) (Phillips and Perron 1988) techniques. Each of these techniques has three different versions: (1) with constant; (2) without constant; and (3) with constant and trend. This paper presents only the results produced from ADF and PP tests using constant and trend. 
However, results from the other two versions of the unit root tests are similar to the third version. Findings from these tests are available upon request.

The standard Johansen $(1991,1995)$ cointegration technique can be applied if all the variables are integrated of order 1 . This method serves as a basis for implementing a vector errorcorrection model (VECM). From this model, the long-run dynamics and the short-run causal relations among the variables can be identified. The Johansen test produces two likelihood ratio statistics, namely the trace and the maximum eigenvalue. Both statistics are reported in this paper. Schwarz's Bayesian information criterion criteria is used to select the optimal lag length. The next stage involves implementing the Granger causality testing within the VECM framework. Granger tests provide the short-run dynamics between changes in the short-term interest rate, Canadian government securities yields, and other control variables.

Table 1: Definition and Source of the Variables

\begin{tabular}{|c|c|c|c|}
\hline $\begin{array}{l}\text { Variable } \\
\text { names }\end{array}$ & Data description & Frequency & Sources \\
\hline \multicolumn{4}{|c|}{ Short-term interest rate } \\
\hline TB3M & $\begin{array}{l}\text { Treasury bills, 3-month, yield, } \% \text {; } \\
\text { 1/4/1990-12/31/2018 }\end{array}$ & Daily & $\begin{array}{l}\text { Bank of Canada; } \\
\text { Macrobond }\end{array}$ \\
\hline \multicolumn{4}{|c|}{ Canadian government security yields } \\
\hline GB2Y & $\begin{array}{l}\text { Canadian government securities, 2-year, yield, \%; } \\
\text { 1/1/1990-12/31/2018 }\end{array}$ & Daily & $\begin{array}{l}\text { Bank of Canada; } \\
\text { Macrobond }\end{array}$ \\
\hline GB3Y & $\begin{array}{l}\text { Canadian government securities, 3-year, yield, \%; } \\
1 / 1 / 1990-12 / 31 / 2018\end{array}$ & Daily & $\begin{array}{l}\text { Bank of Canada; } \\
\text { Macrobond }\end{array}$ \\
\hline GB5Y & $\begin{array}{l}\text { Canadian government securities, 5-year, yield, \%; } \\
\text { 1/1/1990-12/31/2018 }\end{array}$ & Daily & $\begin{array}{l}\text { Bank of Canada; } \\
\text { Macrobond }\end{array}$ \\
\hline GB7Y & $\begin{array}{l}\text { Canadian government securities, 7-year, yield, \%; } \\
\text { 1/1/1990-12/31/2018 }\end{array}$ & Daily & $\begin{array}{l}\text { Bank of Canada; } \\
\text { Macrobond }\end{array}$ \\
\hline GB10Y & $\begin{array}{l}\text { Canadian government securities, } 10 \text {-year, yield, \%; } \\
\text { 1/1/1990-12/31/2018 }\end{array}$ & Daily & $\begin{array}{l}\text { Bank of Canada; } \\
\text { Macrobond }\end{array}$ \\
\hline GB30Y & $\begin{array}{l}\text { Canadian government securities, 30-year, yield, \%; } \\
\text { 1/1/1991-12/31/2018 }\end{array}$ & Daily & $\begin{array}{l}\text { Bank of Canada; } \\
\text { Macrobond }\end{array}$ \\
\hline \multicolumn{4}{|l|}{ Equity } \\
\hline TSX & $\begin{array}{l}\text { S\&P/TSX } 60 \text { equity index, price return, Canadian } \\
\text { dollar, C\$; } \\
\text { 1/1/1990-12/31/2018 }\end{array}$ & Daily & $\begin{array}{l}\text { Toronto Stock Exchange; } \\
\text { Macrobond }\end{array}$ \\
\hline \multicolumn{4}{|c|}{ Energy prices } \\
\hline BRENT & $\begin{array}{l}\text { Crude oil, Brent Europe spot price, freight on board, } \\
\text { US\$; } \\
\text { 1/1/1990-12/31/2018 }\end{array}$ & Daily & $\begin{array}{l}\text { Intercontinental } \\
\text { Exchange; } \\
\text { Macrobond }\end{array}$ \\
\hline \multicolumn{4}{|l|}{ Currency } \\
\hline CAD & $\begin{array}{l}\text { FX spot rate, C\$/US\$; } \\
1 / 1 / 1990-12 / 31 / 2018\end{array}$ & Daily & Macrobond \\
\hline
\end{tabular}




\section{Behavioral Equations}

The following general equation is estimated to examine the relationship between the short-term interest rate and the long-term interest rate on Canadian government securities of various maturity tenors:

$$
\mathrm{GB}=\mathrm{F}^{1}(\mathrm{STIR}, \mathrm{FX}, \mathrm{LN}[\mathrm{EQUITY}], \mathrm{LN}[\mathrm{OIL}])
$$

where GB is the yield on Canadian government securities of different tenors, including 2-year (GB2Y), 3-year (GB3Y), 5-year (GB3Y), 7-year (GB7Y), 10-year (GB10Y), and 30-year (GB30Y) government bonds. The short-term interest rate (STIR) is the yield on Canadian 3month Treasury bills (TB3M). The potential impact of foreign exchange (FX) is represented by the spot rate between the Canadian dollar and US dollar (CAD), measured as Canadian dollar (C\$) per US dollar (US\$). An increase (decrease) in CAD means that the Canadian dollar has depreciated (appreciated) with respect to the US dollar. Brent Europe spot price (BRENT) is used for oil prices (OIL). Standard and Poor's (S\&P) and the Toronto Stock Exchange's (TSX) 60 equity index, which is an index of selected key stock prices listed in Canada's main stock exchange, is used for equity prices (EQUITY). The LN(.) represents the natural logarithmic transformation of selected variables.

Therefore, the behavioral equation estimated in this paper takes the following general form:

$$
\mathrm{GB}_{\mathrm{i}}=\mathrm{F}^{2}(\mathrm{~TB} 3 \mathrm{M}, \mathrm{CAD}, \mathrm{LN}[\mathrm{TSX}], \mathrm{LN}[\mathrm{BRENT}])
$$

where i=2-year, 3-year, 5-year, 7-year, 10-year, and 30-year maturity tenors. Daily data on the relevant variables are used. 


\section{Empirical Findings}

To examine the unit root properties of the variables, both ADF and PP unit root test results with a constant and trend term are reported in table 2. From this table, irrespective of the test used, the results show that the variables are nonstationary at levels, but stationary at first differences.

Table 2: Unit Root Tests

\begin{tabular}{|c|c|c|}
\hline Variable & ADF & $\mathbf{P P}$ \\
\hline GB2Y & -2.797 & -2.698 \\
\hline$\triangle \mathrm{GB} 2 \mathrm{Y}$ & $-80.173 * * *$ & $-80.018 * * *$ \\
\hline GB3Y & -2.913 & -2.891 \\
\hline$\triangle \mathrm{GB} 3 \mathrm{Y}$ & $-80.821^{* * *}$ & $-80.736 * * *$ \\
\hline GB5Y & -2.926 & -2.824 \\
\hline$\triangle \mathrm{GB} 5 \mathrm{Y}$ & $-83.339 * * *$ & $-83.339 * * *$ \\
\hline GB7Y & -2.915 & -2.931 \\
\hline$\Delta \mathrm{GB} 7 \mathrm{Y}$ & $-85.319 * * *$ & $-85.309 * * *$ \\
\hline GB10Y & -3.140 & -3.003 \\
\hline$\Delta \mathrm{GB} 10 \mathrm{Y}$ & $-84.059 * * *$ & $-84.019 * * *$ \\
\hline GB30Y & -3.444 & -3.372 \\
\hline$\Delta \mathrm{GB} 30 \mathrm{Y}$ & $-79.566^{* * *}$ & $-79.367 * * *$ \\
\hline TB3M & -2.808 & -2.787 \\
\hline$\triangle \mathrm{TB} 3 \mathrm{M}$ & $-25.530 * * *$ & $-83.569 * * *$ \\
\hline CAD & -1.642 & -1.514 \\
\hline$\triangle \mathrm{CAD}$ & $-87.213 * * *$ & $-87.546^{* * *}$ \\
\hline $\mathrm{LN}[\mathrm{TSX}]$ & -2.213 & -2.062 \\
\hline$\Delta \mathrm{LN}[\mathrm{TSX}]$ & $-41.190 * * *$ & $-87.319 * * *$ \\
\hline LN[BRENT] & -2.198 & -2.145 \\
\hline$\Delta \mathrm{LN}[\mathrm{BRENT}]$ & $-53.128 * * *$ & $-88.114^{* * *}$ \\
\hline
\end{tabular}

Note: 1) *** indicates statistical significance at the 1 percent level. 2) The null hypothesis of both the ADF and PP tests is that the series contains unit roots.

Table 3 presents results from the Johansen cointegration tests, based on equation (2). The null hypothesis of no cointegration among variables in the model is rejected by both trace and maximum eigenvalue statistics in all six equations. The sequential testing for more than one cointegration equation by both statistics leads to the conclusion that there are two cointegrating vectors in each of the first four equations, and one cointegrating vector in each of the last two equations. Therefore, it is evident that there are long-run cointegrating relationships between government bond yields, short-term interest rates, and other relevant variables. 
Table 3: Johansen Cointegration Tests (equation 2)

\begin{tabular}{|c|c|c|c|}
\hline \multirow{2}{*}{ Hypothesized number of cointegrating equations } & Eigenvalue & Trace statistic & Maximum eigenvalue statistics \\
\hline & \multicolumn{3}{|c|}{$G B 2 Y=F^{2}(T B 3 M, C A D, L N[T S X], L N[B R E N T])$} \\
\hline None & 0.007 & $108.295 * * *$ & $56.134 * * *$ \\
\hline At most 1 & 0.004 & $52.162 * *$ & $33.319 * * *$ \\
\hline At most 2 & 0.002 & 18.844 & 11.771 \\
\hline At most 3 & 0.001 & 7.072 & 5.598 \\
\hline \multirow[t]{2}{*}{ At most 4} & 0.000 & 1.474 & 1.474 \\
\hline & \multicolumn{3}{|c|}{$G B 3 Y=F^{2}(T B 3 M, C A D, L N[T S X], L N[B R E N T])$} \\
\hline None & 0.006 & $96.320 * * *$ & $45.068^{* * *}$ \\
\hline At most 1 & 0.004 & $51.251 * *$ & $32.103 * *$ \\
\hline At most 2 & 0.002 & 19.148 & 11.912 \\
\hline At most 3 & 0.001 & 7.237 & 5.789 \\
\hline \multirow[t]{2}{*}{ At most 4} & 0.000 & 1.447 & 1.447 \\
\hline & \multicolumn{3}{|c|}{$G B 5 Y=F^{2}(T B 3 M, C A D, L N[T S X], L N[B R E N T])$} \\
\hline None & 0.005 & $87.195 * * *$ & $40.910 * * *$ \\
\hline At most 1 & 0.004 & $46.285^{*}$ & $27.481 *$ \\
\hline At most 2 & 0.002 & 18.805 & 11.712 \\
\hline At most 3 & 0.001 & 7.092 & 5.660 \\
\hline \multirow[t]{2}{*}{ At most 4} & 0.000 & 1.432 & 1.432 \\
\hline & \multicolumn{3}{|c|}{$G B 7 Y=F^{2}(T B 3 M, C A D, L N[T S X], L N[B R E N T])$} \\
\hline None & 0.005 & $84.364 * * *$ & $39.734 * * *$ \\
\hline At most 1 & 0.003 & $44.630 *$ & $25.317 *$ \\
\hline At most 2 & 0.002 & 19.313 & 11.943 \\
\hline At most 3 & 0.001 & 7.370 & 5.952 \\
\hline \multirow[t]{2}{*}{ At most 4} & 0.000 & 1.418 & 1.418 \\
\hline & \multicolumn{3}{|c|}{$G B 10 Y=F^{2}(T B 3 M, C A D, L N[T S X], L N[B R E N T])$} \\
\hline None & 0.005 & $84.988 * * *$ & $41.052 * *$ \\
\hline At most 1 & 0.003 & 43.935 & 24.593 \\
\hline At most 2 & 0.002 & 19.342 & 12.088 \\
\hline At most 3 & 0.001 & 7.254 & 5.828 \\
\hline \multirow[t]{2}{*}{ At most 4} & 0.000 & 1.426 & 1.426 \\
\hline & \multicolumn{3}{|c|}{$G B 30 Y=F^{2}(T B 3 M, C A D, L N[T S X], L N[B R E N T])$} \\
\hline None & 0.006 & $85.942 * * *$ & $43.152 * * *$ \\
\hline At most 1 & 0.003 & 42.789 & 21.748 \\
\hline At most 2 & 0.002 & 21.042 & 14.355 \\
\hline At most 3 & 0.001 & 6.686 & 5.214 \\
\hline At most 4 & 0.000 & 1.472 & 1.472 \\
\hline
\end{tabular}

Note: $* * *, * *$, and $*$ indicate statistical significance at the 1 percent, 5 percent, and 10 percent levels, respectively.

To check whether or not the evidence of cointegration found is contingent in the abovementioned model, additional models are constructed as follows:

$$
\begin{aligned}
\mathrm{GB}_{\mathrm{i}} & =\mathrm{F}^{3}(\mathrm{~TB} 3 \mathrm{M}) \\
\mathrm{GB}_{\mathrm{i}} & =\mathrm{F}^{4}(\mathrm{~TB} 3 \mathrm{M}, \mathrm{LN}[\mathrm{TSX}]) \\
\mathrm{GB}_{\mathrm{i}} & =\mathrm{F}^{5}(\mathrm{~TB} 3 \mathrm{M}, \mathrm{CAD}) \\
\mathrm{GB}_{\mathrm{i}} & =\mathrm{F}^{6}(\mathrm{~TB} 3 \mathrm{M}, \mathrm{LN}[\mathrm{BRENT}]) \\
\mathrm{GB}_{\mathrm{i}} & =\mathrm{F}^{7}(\mathrm{~TB} 3 \mathrm{M}, \mathrm{LN}[\mathrm{TSX}], \mathrm{LN}[\mathrm{BRENT}]) \\
\mathrm{GB}_{\mathrm{i}} & =\mathrm{F}^{8}(\mathrm{~TB} 3 \mathrm{M}, \mathrm{LN}[\mathrm{TSX}], \mathrm{CAD}) \\
\mathrm{GB}_{\mathrm{i}} & =\mathrm{F}^{9}(\mathrm{~TB} 3 \mathrm{M}, \mathrm{LN}[\mathrm{BRENT}], \mathrm{CAD})
\end{aligned}
$$


Cointegration tests are carried out for the models above and presented in tables 4-10. Table 4 shows that there is cointegration between government bond yields for all maturities and Treasury bill yields in models where the government bond yields are solely a function of Treasury bill yields. Table 5 shows evidence of cointegration between government bond yields for all maturity tenors, Treasury bill yields, and the log of equity prices. Table 6 shows that there is cointegration between Treasury bill yields, the Canadian dollar, and government bond yields, except for the 7year and 10-year maturity tenors. In table 7, results are presented for the equation where government bond yields are a function of Treasury bills and the log of the crude oil price. Results show that there is evidence of cointegration between government bond yields and other variables, except for bonds of a 10-year maturity tenor. Table 8 presents results from estimating the cointegrating relationships between Treasury bill yields, government bond yields, the log of equity prices, and the log of crude oil prices. The cointegrating relationships are established irrespective of the specific maturity tenors of government bonds used in the equations. Table 9 shows that there is evidence of cointegration between Treasury bill yields, the log of equity prices, the Canadian dollar, and government bond yields of all maturity tenors, except for bonds of a 7-year maturity tenor. Finally, table 10 presents results from estimating the cointegrating relationships between Treasury bill yields, the log of crude oil prices, the Canadian dollar, and government bond yields. The cointegrating relationship is found in four of six equations.

These results, therefore, show that in most cases there is substantial evidence of cointegration in a wide range of models that include government bond yields of various maturity levels, Treasury bill yields, and a range of control variables, such as the log of equity prices, the log of crude oil prices, and the Canadian dollar. 
Table 4: Johansen Cointegration Tests (equation 3)

\begin{tabular}{|c|c|c|c|}
\hline \multirow{2}{*}{ Hypothesized number of cointegrating equations } & Eigenvalue & Trace statistic & Maximum eigenvalue statistics \\
\hline & \multicolumn{3}{|c|}{$G B 2 Y=F^{3}(T B 3 M)$} \\
\hline None & 0.004 & $37.586 * * *$ & $32.474 * * *$ \\
\hline \multirow[t]{2}{*}{ At most 1} & 0.001 & $5.111 * *$ & $5.111 * *$ \\
\hline & \multicolumn{3}{|c|}{$G B 3 Y=F^{3}(T B 3 M)$} \\
\hline None & 0.003 & $30.607 * * *$ & $25.871 * * *$ \\
\hline \multirow{2}{*}{ At most 1} & 0.001 & $4.737 * *$ & $4.737 * *$ \\
\hline & \multicolumn{3}{|c|}{$G B 5 Y=F^{3}(T B 3 M)$} \\
\hline None & 0.003 & $22.908 * * *$ & $19.138 * * *$ \\
\hline \multirow[t]{2}{*}{ At most 1} & 0.000 & $3.769^{*}$ & $3.769^{*}$ \\
\hline & \multicolumn{3}{|c|}{$G B 7 Y=F^{3}(T B 3 M)$} \\
\hline None & 0.002 & $19.678^{* *}$ & $16.118^{* *}$ \\
\hline \multirow[t]{2}{*}{ At most 1} & 0.000 & $3.560^{*}$ & $3.560 *$ \\
\hline & \multicolumn{3}{|c|}{$G B 10 Y=F^{3}(T B 3 M)$} \\
\hline None & 0.002 & $18.465 * *$ & $14.952 * *$ \\
\hline \multirow[t]{2}{*}{ At most 1} & 0.000 & $3.513 *$ & $3.513 *$ \\
\hline & \multicolumn{3}{|c|}{$G B 30 Y=F^{3}(T B 3 M)$} \\
\hline None & 0.002 & $22.033 * * *$ & $18.175^{* *}$ \\
\hline At most 1 & 0.002 & $3.859 * *$ & $3.859 * *$ \\
\hline
\end{tabular}

Note: $* * *, * *$, and $*$ indicate statistical significance at the 1 percent, 5 percent, and 10 percent levels, respectively.

Table 5: Johansen Cointegration Tests (equation 4)

\begin{tabular}{|c|c|c|c|}
\hline \multirow{2}{*}{ Hypothesized number of cointegrating equations } & Eigenvalue & Trace statistic & Maximum eigenvalue statistics \\
\hline & \multicolumn{3}{|c|}{$G B 2 Y=F^{4}(T B 3 M, L N[T S X])$} \\
\hline None & 0.007 & $64.098 * * *$ & $53.324 * * *$ \\
\hline At most 1 & 0.001 & $13.774 *$ & 10.488 \\
\hline \multirow[t]{2}{*}{ At most 2} & 0.000 & $3.286^{*}$ & $3.286^{*}$ \\
\hline & \multicolumn{3}{|c|}{$G B 3 Y=F^{4}(T B 3 M, L N[T S X])$} \\
\hline None & 0.005 & $54.870 * * *$ & $40.983 * * *$ \\
\hline At most 1 & 0.001 & $13.887^{*}$ & 10.523 \\
\hline \multirow[t]{2}{*}{ At most 2} & 0.000 & $3.364 *$ & $3.364 *$ \\
\hline & \multicolumn{3}{|c|}{$G B 5 Y=F^{4}(T B 3 M, L N[T S X])$} \\
\hline None & 0.004 & $45.778 * * *$ & $32.589 * * *$ \\
\hline At most 1 & 0.001 & 13.189 & 9.888 \\
\hline \multirow[t]{2}{*}{ At most 2} & 0.000 & $3.301 *$ & $3.301 *$ \\
\hline & \multicolumn{3}{|c|}{$G B 7 Y=F^{4}(T B 3 M, L N[T S X])$} \\
\hline None & 0.004 & $42.409 * * *$ & $28.898 * * *$ \\
\hline At most 1 & 0.001 & $13.510 *$ & 10.041 \\
\hline \multirow[t]{2}{*}{ At most 2} & 0.000 & $3.469 *$ & $3.469 *$ \\
\hline & \multicolumn{3}{|c|}{$G B 10 Y=F^{4}(T B 3 M, L N[T S X])$} \\
\hline None & 0.004 & $42.153 * * *$ & $28.266^{* * *}$ \\
\hline At most 1 & 0.001 & $13.887^{*}$ & 10.332 \\
\hline \multirow[t]{2}{*}{ At most 2} & 0.000 & $3.555^{*}$ & $3.555^{*}$ \\
\hline & \multicolumn{3}{|c|}{$G B 30 Y=F^{4}(T B 3 M, L N[T S X])$} \\
\hline None & 0.003 & $40.297 * * *$ & $24.098 * *$ \\
\hline At most 1 & 0.002 & $16.1999 * *$ & 11.816 \\
\hline At most 2 & 0.001 & $4.383 * *$ & $4.383 * *$ \\
\hline
\end{tabular}

Note: $* * *, * *$, and $*$ indicate statistical significance at the 1 percent, 5 percent, and 10 percent levels, respectively. 
Table 6: Johansen Cointegration Tests (equation 5)

\begin{tabular}{|c|c|c|c|}
\hline \multirow{2}{*}{ Hypothesized number of cointegrating equations } & Eigenvalue & Trace statistic & Maximum eigenvalue statistics \\
\hline & \multicolumn{3}{|c|}{$G B 2 Y=F^{5}(T B 3 M, C A D)$} \\
\hline None & 0.005 & $48.130 * * *$ & $38.714 * * *$ \\
\hline At most 1 & 0.001 & 9.416 & 7.620 \\
\hline \multirow[t]{2}{*}{ At most 2} & 0.000 & 1.796 & 1.796 \\
\hline & \multicolumn{3}{|c|}{$G B 3 Y=F^{5}(T B 3 M, C A D)$} \\
\hline None & 0.003 & $35.544 * * *$ & $26.224 * * * *$ \\
\hline At most 1 & 0.001 & 9.320 & 7.615 \\
\hline \multirow{2}{*}{ At most 2} & 0.000 & 1.705 & 1.705 \\
\hline & \multicolumn{3}{|c|}{$G B 5 Y=F^{5}(T B 3 M, C A D)$} \\
\hline None & 0.003 & $27.404^{*}$ & $49.271^{*}$ \\
\hline At most 1 & 0.001 & 8.133 & 6.580 \\
\hline \multirow[t]{2}{*}{ At most 2} & 0.000 & 1.553 & 1.553 \\
\hline & \multicolumn{3}{|c|}{$G B 7 Y=F^{5}(T B 3 M, C A D)$} \\
\hline None & 0.002 & 24.132 & 16.279 \\
\hline At most 1 & 0.001 & 7.853 & 6.382 \\
\hline \multirow[t]{2}{*}{ At most 2} & 0.000 & 1.471 & 1.471 \\
\hline & \multicolumn{3}{|c|}{$G B 10 Y=F^{5}(T B 3 M, C A D)$} \\
\hline None & 0.002 & 22.822 & 15.319 \\
\hline At most 1 & 0.001 & 7.502 & 6.031 \\
\hline \multirow[t]{2}{*}{ At most 2} & 0.000 & 1.472 & 1.472 \\
\hline & \multicolumn{3}{|c|}{$G B 30 Y=F^{5}(T B 3 M, C A D)$} \\
\hline None & 0.003 & $28.386^{*}$ & $21.448^{* *}$ \\
\hline At most 1 & 0.001 & 6.937 & 5.472 \\
\hline At most 2 & 0.000 & 1.466 & 1.466 \\
\hline
\end{tabular}

Note: $* * * * *$, and $*$ indicate statistical significance at the 1 percent, 5 percent, and 10 percent level, respectively.

Table 7: Johansen Cointegration Tests (equation 6)

\begin{tabular}{|c|c|c|c|}
\hline \multirow{2}{*}{ Hypothesized number of cointegrating equations } & Eigenvalue & Trace statistic & Maximum eigenvalue statistics \\
\hline & \multicolumn{3}{|c|}{$G B 2 Y=F^{6}(T B 3 M, L N[B R E N T])$} \\
\hline None & 0.006 & $55.971 * * *$ & $43.341 * * *$ \\
\hline At most 1 & 0.001 & 12.630 & 10.547 \\
\hline \multirow[t]{2}{*}{ At most 2} & 0.000 & 2.083 & 2.083 \\
\hline & \multicolumn{3}{|c|}{$G B 3 Y=F^{6}(T B 3 M, L N[B R E N T])$} \\
\hline None & 0.004 & $41.841 * * *$ & $28.904 * * *$ \\
\hline At most 1 & 0.001 & 12.938 & 10.892 \\
\hline \multirow[t]{2}{*}{ At most 2} & 0.000 & 2.046 & 2.046 \\
\hline & \multicolumn{3}{|c|}{$G B 5 Y=F^{6}(T B 3 M, L N[B R E N T])$} \\
\hline None & 0.003 & $31.948 * *$ & $20.087^{*}$ \\
\hline At most 1 & 0.001 & 11.861 & 9.887 \\
\hline \multirow[t]{2}{*}{ At most 2} & 0.000 & 1.974 & 1.974 \\
\hline & \multicolumn{3}{|c|}{$G B 7 Y=F^{6}(T B 3 M, L N[B R E N T])$} \\
\hline None & 0.002 & $28.088^{*}$ & 16.333 \\
\hline At most 1 & 0.001 & 11.754 & 9.822 \\
\hline \multirow[t]{2}{*}{ At most 2} & 0.000 & 1.932 & 1.932 \\
\hline & \multicolumn{3}{|c|}{$G B 10 Y=F^{6}(T B 3 M, L N[B R E N T])$} \\
\hline None & 0.002 & 26.112 & 14.985 \\
\hline At most 1 & 0.001 & 11.128 & 9.211 \\
\hline \multirow[t]{2}{*}{ At most 2} & 0.000 & 1.917 & 1.917 \\
\hline & \multicolumn{3}{|c|}{$G B 30 Y=F^{6}(T B 3 M, L N[B R E N T])$} \\
\hline None & 0.003 & $31.738 * *$ & $22.902 * *$ \\
\hline At most 1 & 0.001 & 8.836 & 6.673 \\
\hline At most 2 & 0.000 & 2.163 & 2.163 \\
\hline
\end{tabular}

Note: $* * *, * *$, and $*$ indicate statistical significance at the 1 percent, 5 percent, and 10 percent level, respectively. 
Table 8: Johansen Cointegration Tests (equation 7)

\begin{tabular}{|c|c|c|c|}
\hline \multirow{2}{*}{ Hypothesized number of cointegrating equations } & Eigenvalue & Trace statistic & Maximum eigenvalue statistics \\
\hline & \multicolumn{3}{|c|}{$G B 2 Y=F^{7}(T B 3 M, L N[T S X], L N[B R E N T])$} \\
\hline None & 0.007 & $79.456 * * *$ & $54.729 * * *$ \\
\hline At most 1 & 0.002 & 24.727 & 14.521 \\
\hline At most 2 & 0.001 & 10.206 & 7.695 \\
\hline \multirow[t]{2}{*}{ At most 3} & 0.000 & 2.511 & 2.511 \\
\hline & \multicolumn{3}{|c|}{$G B 3 Y=F^{7}(T B 3 M, L N[T S X], L N[B R E N T])$} \\
\hline None & 0.006 & $67.534 * * *$ & $42.483 * * *$ \\
\hline At most 1 & 0.002 & 25.051 & 14.964 \\
\hline At most 2 & 0.001 & 10.087 & 7.548 \\
\hline \multirow[t]{2}{*}{ At most 3} & 0.000 & 2.538 & 2.538 \\
\hline & \multicolumn{3}{|c|}{$G B 5 Y=F^{7}(T B 3 M, L N[T S X], L N[B R E N T])$} \\
\hline None & 0.005 & $58.562 * * *$ & $34.917 * * *$ \\
\hline At most 1 & 0.002 & 23.645 & 13.641 \\
\hline At most 2 & 0.001 & 10.003 & 7.414 \\
\hline \multirow[t]{2}{*}{ At most 3} & 0.000 & 2.589 & 2.589 \\
\hline & \multicolumn{3}{|c|}{$G B 7 Y=F^{7}(T B 3 M, L N[T S X], L N[B R E N T])$} \\
\hline None & 0.004 & $55.789 * * *$ & $31.735^{* *}$ \\
\hline At most 1 & 0.002 & 24.053 & 13.862 \\
\hline At most 2 & 0.001 & 10.192 & 7.541 \\
\hline \multirow[t]{2}{*}{ At most 3} & 0.000 & 2.650 & 2.650 \\
\hline & \multicolumn{3}{|c|}{$G B 10 Y=F^{7}(T B 3 M, L N[T S X], L N[B R E N T])$} \\
\hline None & 0.004 & $56.240 * * *$ & $32.425^{* *}$ \\
\hline At most 1 & 0.002 & 23.815 & 13.820 \\
\hline At most 2 & 0.001 & 9.995 & 7.297 \\
\hline \multirow[t]{2}{*}{ At most 3} & 0.000 & 2.698 & 2.698 \\
\hline & \multicolumn{3}{|c|}{$G B 30 Y=F^{7}(T B 3 M, L N[T S X], L N[B R E N T])$} \\
\hline None & 0.005 & $58.076^{* * *}$ & $34.058 * * *$ \\
\hline At most 1 & 0.002 & 24.018 & 15.393 \\
\hline At most 2 & 0.001 & 8.624 & 5.207 \\
\hline At most 3 & 0.000 & $3.418^{*}$ & $3.418^{*}$ \\
\hline
\end{tabular}

Note: ${ }^{* *},{ }^{* *}$, and $*$ indicate statistical significance at the 1 percent, 5 percent, and 10 percent level respectively. 
Table 9: Johansen Cointegration Tests (equation 8)

\begin{tabular}{|c|c|c|c|}
\hline \multirow{2}{*}{ Hypothesized number of cointegrating equations } & Eigenvalue & Trace statistic & Maximum eigenvalue statistics \\
\hline & \multicolumn{3}{|c|}{$G B 2 Y=F^{8}(T B 3 M, L N[T S X], C A D)$} \\
\hline None & 0.007 & $73.943 * * *$ & $54.753 * * *$ \\
\hline At most 1 & 0.002 & 19.190 & 11.859 \\
\hline At most 2 & 0.001 & 7.331 & 5.709 \\
\hline \multirow[t]{2}{*}{ At most 3} & 0.000 & 1.622 & 1.622 \\
\hline & \multicolumn{3}{|c|}{$G B 3 Y=F^{8}(T B 3 M, L N[T S X], C A D)$} \\
\hline None & 0.005 & $61.058 * * *$ & $41.627 * * *$ \\
\hline At most 1 & 0.002 & 19.431 & 11.907 \\
\hline At most 2 & 0.001 & 7.524 & 5.943 \\
\hline \multirow[t]{2}{*}{ At most 3} & 0.000 & 1.581 & 1.581 \\
\hline & \multicolumn{3}{|c|}{$G B 5 Y=F^{8}(T B 3 M, L N[T S X], C A D)$} \\
\hline None & 0.004 & $51.951 * *$ & $33.346 * * *$ \\
\hline At most 1 & 0.001 & 18.605 & 11.222 \\
\hline At most 2 & 0.001 & 7.383 & 5.863 \\
\hline \multirow[t]{2}{*}{ At most 3} & 0.000 & 1.520 & 1.520 \\
\hline & \multicolumn{3}{|c|}{$G B 7 Y=F^{8}(T B 3 M, L N[T S X], C A D)$} \\
\hline None & 0.004 & 48.405 & 29.472 \\
\hline At most 1 & 0.001 & 18.933 & 11.290 \\
\hline At most 2 & 0.001 & 7.643 & 6.144 \\
\hline \multirow[t]{2}{*}{ At most 3} & 0.000 & 1.499 & 1.499 \\
\hline & \multicolumn{3}{|c|}{$G B 10 Y=F^{8}(T B 3 M, L N[T S X], C A D)$} \\
\hline None & 0.004 & $48.388^{* *}$ & $29.311 * *$ \\
\hline At most 1 & 0.002 & 19.077 & 11.542 \\
\hline At most 2 & 0.001 & 7.535 & 6.019 \\
\hline \multirow[t]{2}{*}{ At most 3} & 0.000 & 1.516 & 1.516 \\
\hline & \multicolumn{3}{|c|}{$G B 30 Y=F^{8}(T B 3 M, L N[T S X], C A D)$} \\
\hline None & 0.004 & $48.445^{* *}$ & $27.775^{* *}$ \\
\hline At most 1 & 0.002 & 20.670 & 13.203 \\
\hline At most 2 & 0.001 & 7.467 & 5.891 \\
\hline At most 3 & 0.000 & 1.575 & 1.575 \\
\hline
\end{tabular}

Note: ${ }^{* *},{ }^{* *}$, and $*$ indicate statistical significance at the 1 percent, 5 percent, and 10 percent level, respectively. 
Table 10: Johansen Cointegration Tests (equation 9)

\begin{tabular}{|c|c|c|c|}
\hline \multirow{2}{*}{ Hypothesized number of cointegrating equations } & Eigenvalue & Trace statistic & Maximum eigenvalue statistics \\
\hline & \multicolumn{3}{|c|}{$G B 2 Y=F^{9}(T B 3 M, L N[B R E N T], C A D)$} \\
\hline None & 0.006 & $70.862 * * *$ & $46.764 * * *$ \\
\hline At most 1 & 0.002 & 24.098 & 15.220 \\
\hline At most 2 & 0.001 & 8.878 & 7.5217 \\
\hline \multirow[t]{2}{*}{ At most 3} & 0.000 & 1.356 & 1.356 \\
\hline & \multicolumn{3}{|c|}{$G B 3 Y=F^{9}(T B 3 M, L N[B R E N T], C A D)$} \\
\hline None & 0.004 & $57.167 * * *$ & $32.927 * * *$ \\
\hline At most 1 & 0.002 & 24.239 & 15.315 \\
\hline At most 2 & 0.001 & 8.924 & 7.608 \\
\hline \multirow[t]{2}{*}{ At most 3} & 0.000 & 1.316 & 1.316 \\
\hline & \multicolumn{3}{|c|}{$G B 5 Y=F^{9}(T B 3 M, L N[B R E N T], C A D)$} \\
\hline None & 0.003 & $47.365^{*}$ & 24.898 \\
\hline At most 1 & 0.002 & 22.467 & 14.383 \\
\hline At most 2 & 0.001 & 8.083 & 6.810 \\
\hline \multirow[t]{2}{*}{ At most 3} & 0.000 & 1.273 & 1.273 \\
\hline & \multicolumn{3}{|c|}{$G B 7 Y=F^{9}(T B 3 M, L N[B R E N T], C A D)$} \\
\hline None & 0.003 & 43.656 & 21.717 \\
\hline At most 1 & 0.002 & 21.940 & 14.072 \\
\hline At most 2 & 0.001 & 7.867 & 6.633 \\
\hline \multirow[t]{2}{*}{ At most 3} & 0.000 & 1.234 & 1.234 \\
\hline & \multicolumn{3}{|c|}{$G B 10 Y=F^{9}(T B 3 M, L N[B R E N T], C A D)$} \\
\hline None & 0.003 & 41.934 & 20.585 \\
\hline At most 1 & 0.002 & 21.350 & 13.891 \\
\hline At most 2 & 0.001 & 7.459 & 6.233 \\
\hline \multirow[t]{2}{*}{ At most 3} & 0.000 & 1.226 & 1.226 \\
\hline & \multicolumn{3}{|c|}{$G B 30 Y=F^{9}(T B 3 M, L N[B R E N T], C A D)$} \\
\hline None & 0.003 & $47.914 * *$ & 23.979 \\
\hline At most 1 & 0.002 & 23.935 & 17.038 \\
\hline At most 2 & 0.001 & 6.897 & 5.639 \\
\hline At most 3 & 0.000 & 1.258 & 1.258 \\
\hline
\end{tabular}

Note: $* * *, * *$, and $*$ indicate statistical significance at the 1 percent, 5 percent, and 10 percent level, respectively.

The next set of tables (11-16) present causality results based on the first model (equation 2). The causal ordering is established by applying the Granger causality test (that is, block exogeneity Wald test) within the VECM framework. These results indicate that in the short run, $\triangle T B 3 \mathrm{M}$ Granger causes $\Delta \mathrm{GB} 2 \mathrm{Y}, \Delta \mathrm{GB} 3 \mathrm{Y}, \Delta \mathrm{GB} 5 \mathrm{Y}$, and $\Delta \mathrm{GB} 7 \mathrm{Y}$. This effect dissipates when $\Delta \mathrm{GB} 10 \mathrm{Y}$ and $\triangle \mathrm{GB} 30 \mathrm{Y}$ are used as dependent variables. $\triangle \mathrm{GBs}$ consistently Granger causes $\triangle \mathrm{TB} 3 \mathrm{M}$ when the latter is included in the equation as the dependent variable. Therefore, it can be argued that the daily change in the short-term interest rate generally has an impact on the daily change in long-term bond yields with a maturity of 7 years or less. However, this impact disappears when the tenor of long-term bonds is 10 years and above. Long-term government bonds, however, consistently Granger cause the short-term interest rate. Therefore, in most cases, there is evidence of bidirectional causality between long-term bond yields and the short-term interest rate. Among other variables, there is evidence of bidirectional Granger causality between $\Delta \mathrm{GBs}$ 
and $\triangle \mathrm{LN}[\mathrm{TSX}]$, unidirectional causality from $\triangle \mathrm{GBs}$ to $\triangle \mathrm{CAD}$, and no Granger causality (with the exception of the GB10Y equation) between $\triangle \mathrm{GBs}$ and $\triangle \mathrm{LN}[\mathrm{BRENT}]$.

Table 11: Granger Causality within the VECM Framework (equation 2: $\triangle$ GB2Y)

\begin{tabular}{lccccc}
\hline & \multicolumn{5}{c}{ Dependent Variable } \\
\cline { 2 - 6 } Excluded & $\Delta \mathrm{GB} 2 \mathrm{Y}$ & $\Delta \mathrm{TB} 3 \mathrm{M}$ & $\Delta \mathrm{CAD}$ & $\Delta \mathrm{LN}[\mathrm{TSX}]$ & $\Delta \mathrm{LN}[\mathrm{BRENT}]$ \\
\hline$\Delta \mathrm{GB} 2 \mathrm{Y}$ & - & $610.904^{* * *}$ & $10.947^{* * *}$ & $14.375^{* * *}$ & 2.861 \\
$\Delta \mathrm{TB} 3 \mathrm{M}$ & $12.927^{* * *}$ & - & 3.238 & $7.404^{* *}$ & 3.456 \\
$\Delta \mathrm{CAD}$ & 0.932 & $5.929^{*}$ & - & $19.603^{* * *}$ & $96.721^{* * *}$ \\
$\Delta \mathrm{LN}[\mathrm{TSX}]$ & $9.409^{* * *}$ & $9.344^{* * *}$ & $180.028^{* * *}$ & - & $64.928^{* * *}$ \\
$\Delta \mathrm{LN}[\mathrm{BRENT}]$ & 0.021 & 1.808 & $9.204^{* *}$ & 0.750 & - \\
\hline All & $23.898^{* * *}$ & $627.197^{* * *}$ & $208.116^{* * *}$ & $53.073^{* * *}$ & 221.483 \\
\hline
\end{tabular}

Note: $* * *$ and $* *$ indicate statistical significance at the 1 percent and 5 percent levels, respectively.

Table 12: Granger Causality within the VECM Framework (equation 2: $\triangle$ GB3Y)

\begin{tabular}{lccccc}
\hline & \multicolumn{3}{c}{ Dependent Variable } \\
\cline { 2 - 5 } Excluded & $\Delta \mathrm{GB} 3 \mathrm{Y}$ & $\Delta \mathrm{TB} 3 \mathrm{M}$ & $\Delta \mathrm{CAD}$ & $\Delta \mathrm{LN}[\mathrm{TSX}]$ & $\Delta \mathrm{LN}[\mathrm{BRENT}]$ \\
\hline$\Delta \mathrm{GB} 3 \mathrm{Y}$ & - & $437.504^{* * *}$ & $11.798^{* * *}$ & $14.428^{* * *}$ & 1.659 \\
$\Delta \mathrm{TB} 3 \mathrm{M}$ & $6.683^{* *}$ & - & 2.456 & $9.077^{* *}$ & 2.925 \\
$\Delta \mathrm{CAD}$ & 3.008 & $6.584^{* *}$ & - & $19.684^{* * *}$ & $95.851^{* * *}$ \\
$\Delta \mathrm{LN}[\mathrm{TSX}]$ & $10.000^{* * *}$ & $8.395^{* *}$ & $178.159^{* * *}$ & - & $65.047^{* * *}$ \\
$\Delta \mathrm{LN}[\mathrm{BRENT}]$ & 0.851 & 1.525 & $9.122^{* *}$ & 0.810 & - \\
\hline All & $20.516^{* * *}$ & $453.419^{* * *}$ & $208.918^{* * *}$ & $53.163^{* * *}$ & $220.136^{* * *}$ \\
\hline
\end{tabular}

Note: $* * *$ and $* *$ indicate statistical significance at the 1 percent and 5 percent levels, respectively.

Table 13: Granger Causality within the VECM Framework (equation 2: $\triangle$ GB5Y)

\begin{tabular}{lccccc}
\hline & \multicolumn{5}{c}{ Dependent Variable } \\
\cline { 2 - 6 } Excluded & $\Delta \mathrm{GB} 5 \mathrm{Y}$ & $\Delta \mathrm{TB} 3 \mathrm{M}$ & $\Delta \mathrm{CAD}$ & $\Delta \mathrm{LN}[\mathrm{TSX}]$ & $\Delta \mathrm{LN}[\mathrm{BRENT}]$ \\
\hline$\Delta$ GB5Y & - & $383.964^{* * *}$ & $14.492^{* * *}$ & $18.377^{* * *}$ & 3.033 \\
$\Delta \mathrm{TB} 3 \mathrm{M}$ & $9.028^{* *}$ & - & 2.477 & $9.545^{* * *}$ & 4.189 \\
$\Delta \mathrm{CAD}$ & 0.547 & $7.966^{* *}$ & - & $20.018^{* * *}$ & $96.361^{* * *}$ \\
$\Delta \mathrm{LN}[\mathrm{TSX}]$ & $12.740^{* * *}$ & $10.371^{* * *}$ & $175.695^{* * *}$ & - & $64.494^{* * *}$ \\
$\Delta \mathrm{LN}[\mathrm{BRENT}]$ & 0.318 & 1.406 & $8.692^{* *}$ & 0.881 & - \\
\hline All & $22.874 * * *$ & $400.211^{* * *}$ & $211.592^{* * *}$ & $57.236^{* * *}$ & $220.680^{* * *}$ \\
\hline
\end{tabular}

Note: $* * *$ and $* *$ indicate statistical significance at the 1 percent and 5 percent levels, respectively.

Table 14: Granger Causality within the VECM Framework (equation 2: $\triangle$ GB7Y)

\begin{tabular}{lccccc}
\hline & \multicolumn{5}{c}{ Dependent Variable } \\
\cline { 2 - 6 } Excluded & $\Delta \mathrm{GB} 7 \mathrm{Y}$ & $\Delta \mathrm{TB} 3 \mathrm{M}$ & $\Delta \mathrm{CAD}$ & $\Delta \mathrm{LN}[\mathrm{TSX}]$ & $\Delta \mathrm{LN}[\mathrm{BRENT}]$ \\
\hline$\Delta$ GB7Y & - & $280.816^{* * *}$ & $9.255^{* * *}$ & $22.406^{* * *}$ & 3.591 \\
$\Delta \mathrm{TB} 3 \mathrm{M}$ & $8.363^{* *}$ & - & 1.850 & $9.344^{* * *}$ & 4.102 \\
$\Delta \mathrm{CAD}$ & 0.024 & $9.398^{* * *}$ & - & $20.981^{* * *}$ & $95.745^{* * *}$ \\
$\Delta \mathrm{LN}[\mathrm{TSX}]$ & $14.625^{* * *}$ & $8.873^{* *}$ & $176.300^{* * *}$ & - & $64.232^{* * *}$ \\
$\Delta \mathrm{LN}[\mathrm{BRENT}]$ & 0.430 & 1.422 & $8.804^{* *}$ & 0.988 & - \\
\hline All & $24.130^{* * *}$ & $296.900^{* * *}$ & $206.301^{* * *}$ & $61.284^{* * *}$ & $220.832^{* * *}$ \\
\hline
\end{tabular}

Note: $* * *$ and $* *$ indicate statistical significance at the 1 percent and 5 percent levels, respectively. 
Table 15: Granger Causality within the VECM Framework (equation 2: $\triangle$ GB10Y)

\begin{tabular}{lccccc}
\hline & \multicolumn{3}{c}{ Dependent Variable } \\
\cline { 2 - 5 } Excluded & $\Delta \mathrm{GB} 10 \mathrm{Y}$ & $\Delta \mathrm{TB} 3 \mathrm{M}$ & $\Delta \mathrm{CAD}$ & $\Delta \mathrm{LN}[\mathrm{TSX}]$ & $\Delta \mathrm{LN}[\mathrm{BRENT}]$ \\
\hline$\Delta \mathrm{GB} 10 \mathrm{Y}$ & - & $234.761^{* * *}$ & $13.624^{* * *}$ & $24.447^{* * *}$ & $10.420^{* * *}$ \\
$\Delta \mathrm{TB} 3 \mathrm{M}$ & 2.604 & - & 1.635 & $10.477^{* * *}$ & 3.757 \\
$\Delta \mathrm{CAD}$ & 0.397 & $8.260^{* *}$ & - & $20.861^{* * *}$ & $96.648^{* * *}$ \\
$\Delta \mathrm{LN}[\mathrm{TSX}]$ & $11.791^{* * *}$ & $8.900^{* *}$ & $174.307 * * *$ & - & $63.650^{* * *}$ \\
$\Delta \mathrm{LN}[\mathrm{BRENT}]$ & 0.642 & 1.793 & $8.844^{* *}$ & 0.788 & - \\
\hline All & $15.519^{* *}$ & $251.726^{* * *}$ & $210.790^{* * *}$ & $63.845^{* * *}$ & $228.373^{* * *}$ \\
\hline
\end{tabular}

Note: $* * *$ and $* *$ indicate statistical significance at the 1 percent and 5 percent levels, respectively.

Table 16: Granger Causality within the VECM Framework (equation 2: $\triangle$ GB30Y)

\begin{tabular}{lccccc}
\hline & \multicolumn{5}{c}{ Dependent Variable } \\
\cline { 2 - 6 } Excluded & $\Delta \mathrm{GB} 30 \mathrm{Y}$ & $\Delta \mathrm{TB} 3 \mathrm{M}$ & $\Delta \mathrm{CAD}$ & $\Delta \mathrm{LN}[\mathrm{TSX}]$ & $\Delta \mathrm{LN}[\mathrm{BRENT}]$ \\
\hline$\Delta \mathrm{GB} 30 \mathrm{Y}$ & - & $193.012^{* * *}$ & $6.120^{* *}$ & $15.873^{* * *}$ & 4.488 \\
$\Delta \mathrm{TB} 3 \mathrm{M}$ & 2.077 & - & 1.860 & $12.102^{* * *}$ & $4.772^{*}$ \\
$\Delta \mathrm{CAD}$ & 0.040 & $7.820^{* *}$ & - & $21.309^{* * *}$ & $103.504^{* * *}$ \\
$\Delta \mathrm{LN}[\mathrm{TSX}]$ & $4.901^{*}$ & $7.454^{* *}$ & $175.774^{* * *}$ & - & $74.553^{* * *}$ \\
$\Delta \mathrm{LN}[\mathrm{BRENT}]$ & 1.915 & 3.307 & $10.921^{* * *}$ & 1.636 & - \\
\hline All & 9.213 & $209.095^{* * *}$ & $200.156^{* * *}$ & $55.550^{* * *}$ & $249.446^{* * *}$ \\
\hline
\end{tabular}

Note: $* * *, * *$, and $*$ indicate statistical significance at the 1 percent, 5 percent, and 10 percent levels, respectively.

The last set of tables (17-23) present Granger causality results from estimating equations 3-9. These results reinforce the earlier findings from equation 2. In most cases, $\triangle$ TB3M Granger causes $\Delta \mathrm{GB}_{\mathrm{i}}$; in all cases $\Delta \mathrm{GB}_{\mathrm{i}}$ Granger causes $\Delta \mathrm{TB} 3 \mathrm{M}$. Therefore, there is general evidence of bidirectional Granger causality between $\triangle \mathrm{TB} 3 \mathrm{M}$ and $\Delta \mathrm{GB}_{\mathrm{i}}$. The other bidirectional Granger relationship that is generally evident in these findings is the connection between the change in bond yields and the change in the log of the equity index: $\Delta \mathrm{GB}_{\mathrm{i}}$ and $\Delta \mathrm{LN}[\mathrm{TSX}]$. It should be recognized that the findings related to Granger causality merely establish statistical evidence of temporal precedence, not definitive provenience. 
Table 17: Granger Causality within the VECM Framework (equation 3)

\begin{tabular}{|c|c|c|}
\hline \multirow[b]{2}{*}{ Excluded } & \multicolumn{2}{|c|}{ Dependent Variable } \\
\hline & $\Delta \mathrm{GB} 2 \mathrm{Y}$ & $\Delta \mathrm{TB} 3 \mathrm{M}$ \\
\hline$\Delta \mathrm{GB} 2 \mathrm{Y}$ & - & $631.692 * * *$ \\
\hline$\Delta \mathrm{TB} 3 \mathrm{M}$ & $15.921 * * *$ & - \\
\hline \multirow[t]{2}{*}{ All } & $15.921 * * *$ & $631.692 * * *$ \\
\hline & $\Delta$ GB3Y & $\Delta \mathrm{TB} 3 \mathrm{M}$ \\
\hline$\Delta \mathrm{GB} 3 \mathrm{Y}$ & - & $438.330 * * *$ \\
\hline$\triangle \mathrm{TB} 3 \mathrm{M}$ & $6.504 * *$ & - \\
\hline \multirow[t]{2}{*}{ All } & $6.504 * *$ & $438.330 * * *$ \\
\hline & $\Delta \mathrm{GB} 5 \mathrm{Y}$ & $\Delta \mathrm{TB} 3 \mathrm{M}$ \\
\hline$\Delta \mathrm{GB} 5 \mathrm{Y}$ & - & $380.662 * * *$ \\
\hline$\Delta \mathrm{TB} 5 \mathrm{M}$ & $8.858 * *$ & - \\
\hline \multirow[t]{2}{*}{ All } & $8.858 * *$ & $380.662 * * *$ \\
\hline & $\Delta \mathrm{GB} 7 \mathrm{Y}$ & $\Delta \mathrm{TB} 3 \mathrm{M}$ \\
\hline$\Delta \mathrm{GB} 7 \mathrm{Y}$ & - & $277.319 * * *$ \\
\hline$\Delta \mathrm{TB} 7 \mathrm{M}$ & $8.538 * *$ & - \\
\hline \multirow[t]{2}{*}{ All } & $8.538 * *$ & $277.319 * * *$ \\
\hline & $\Delta \mathrm{GB} 10 \mathrm{Y}$ & $\Delta \mathrm{TB} 3 \mathrm{M}$ \\
\hline$\Delta \mathrm{GB} 10 \mathrm{Y}$ & - & $230.690 * * *$ \\
\hline$\Delta \mathrm{TB} 10 \mathrm{M}$ & 2.732 & - \\
\hline \multirow[t]{2}{*}{ All } & 2.732 & $230.690 * * *$ \\
\hline & $\Delta \mathrm{GB} 30 \mathrm{Y}$ & $\Delta \mathrm{TB} 3 \mathrm{M}$ \\
\hline$\Delta \mathrm{GB} 30 \mathrm{Y}$ & - & $188.261 * * *$ \\
\hline$\Delta \mathrm{TB} 10 \mathrm{M}$ & 2.119 & - \\
\hline All & 2.119 & $188.261 * * *$ \\
\hline
\end{tabular}

Note: $* * *$ and $* *$ indicate statistical significance at the 1 percent and 5 percent levels, respectively. 
Table 18: Granger Causality within the VECM Framework (equation 4)

\begin{tabular}{|c|c|c|c|}
\hline \multirow[b]{2}{*}{ Excluded } & \multicolumn{3}{|c|}{ Dependent Variable } \\
\hline & $\Delta \mathrm{GB} 2 \mathrm{Y}$ & $\Delta \mathrm{TB} 3 \mathrm{M}$ & $\Delta \mathrm{LN}[\mathrm{TSX}]$ \\
\hline$\Delta \mathrm{GB} 2 \mathrm{Y}$ & - & $613.937 * * *$ & $14.987 * * *$ \\
\hline$\triangle \mathrm{TB} 3 \mathrm{M}$ & $12.616^{* * *}$ & - & $7.877 * * *$ \\
\hline$\Delta \mathrm{LN}[\mathrm{TSX}]$ & $9.459 * * *$ & $12.794 * * *$ & - \\
\hline \multirow[t]{2}{*}{ All } & $22.345^{* * *}$ & $619.851 * * *$ & $32.988 * * *$ \\
\hline & $\Delta \mathrm{GB} 3 \mathrm{Y}$ & $\Delta \mathrm{TB} 3 \mathrm{M}$ & $\Delta \mathrm{LN}[\mathrm{TSX}]$ \\
\hline$\Delta \mathrm{GB} 3 \mathrm{Y}$ & - & $439.634 * * *$ & $14.857 * * *$ \\
\hline$\Delta \mathrm{TB} 3 \mathrm{M}$ & $6.169 * *$ & - & $9.606 * * *$ \\
\hline$\Delta \mathrm{LN}[\mathrm{TSX}]$ & $9.231 * * *$ & $11.732 * * *$ & - \\
\hline \multirow[t]{2}{*}{ All } & $15.735^{* * *}$ & $445.363 * * *$ & $32.881 * * *$ \\
\hline & $\Delta \mathrm{GB} 5 \mathrm{Y}$ & $\Delta \mathrm{TB} 3 \mathrm{M}$ & $\Delta \mathrm{LN}[\mathrm{TSX}]$ \\
\hline$\Delta \mathrm{GB} 5 \mathrm{Y}$ & - & $384.834 * * *$ & $18.449 * * *$ \\
\hline$\triangle \mathrm{TB} 3 \mathrm{M}$ & $8.715^{* *}$ & - & $10.161 * * *$ \\
\hline$\Delta \mathrm{LN}[\mathrm{TSX}]$ & $12.580^{* * *}$ & $14.442 * * *$ & - \\
\hline \multirow[t]{2}{*}{ All } & $21.575^{* * *}$ & $390.665^{* * *}$ & $36.621 * * *$ \\
\hline & $\Delta \mathrm{GB} 7 \mathrm{Y}$ & $\Delta \mathrm{TB} 3 \mathrm{M}$ & $\Delta \mathrm{LN}[\mathrm{TSX}]$ \\
\hline$\Delta \mathrm{GB} 7 \mathrm{Y}$ & - & $280.232 * * *$ & $21.476^{* * *}$ \\
\hline$\triangle \mathrm{TB} 3 \mathrm{M}$ & $8.357 * *$ & - & $9.949^{* * *}$ \\
\hline$\Delta \mathrm{LN}[\mathrm{TSX}]$ & $14.588^{* * *}$ & $12.970 * * *$ & - \\
\hline \multirow[t]{2}{*}{ All } & $23.281 * * *$ & $285.978 * * *$ & $39.638 * * *$ \\
\hline & $\Delta \mathrm{GB} 10 \mathrm{Y}$ & $\Delta \mathrm{TB} 3 \mathrm{M}$ & $\Delta \mathrm{LN}[\mathrm{TSX}]$ \\
\hline$\Delta \mathrm{GB} 10 \mathrm{Y}$ & - & $233.853 * * *$ & $23.001 * * *$ \\
\hline$\triangle \mathrm{TB} 3 \mathrm{M}$ & 2.585 & - & $10.877 * * *$ \\
\hline$\Delta \mathrm{LN}[\mathrm{TSX}]$ & $11.540^{* * *}$ & $12.367 * * *$ & - \\
\hline \multirow[t]{2}{*}{ All } & $14.397 * * *$ & $239.559 * * *$ & $41.117 * * *$ \\
\hline & $\Delta \mathrm{GB} 30 \mathrm{Y}$ & $\Delta \mathrm{TB} 3 \mathrm{M}$ & $\Delta \mathrm{LN}[\mathrm{TSX}]$ \\
\hline$\Delta \mathrm{GB} 30 \mathrm{Y}$ & - & $190.878^{* * *}$ & $14.111 * * *$ \\
\hline$\triangle \mathrm{TB} 3 \mathrm{M}$ & 2.103 & - & $12.712 * * *$ \\
\hline$\Delta \mathrm{LN}[\mathrm{TSX}]$ & $5.087 *$ & $10.222 * * *$ & - \\
\hline All & 7.215 & $196.359 * * *$ & $31.935 * * *$ \\
\hline
\end{tabular}

Note: $* * *, * *$, and $*$ indicate statistical significance at the 1 percent, 5 percent, and 10 percent levels, respectively. 
Table 19: Granger Causality within the VECM Framework (equation 5)

\begin{tabular}{|c|c|c|c|}
\hline \multirow[b]{2}{*}{ Excluded } & \multicolumn{3}{|c|}{ Dependent Variable } \\
\hline & $\Delta \mathrm{GB} 2 \mathrm{Y}$ & $\Delta \mathrm{TB} 3 \mathrm{M}$ & $\triangle \mathrm{CAD}$ \\
\hline$\Delta \mathrm{GB} 2 \mathrm{Y}$ & - & $611.191 * * *$ & $13.465 * * *$ \\
\hline$\Delta \mathrm{TB} 3 \mathrm{M}$ & $13.072 * * *$ & - & 4.241 \\
\hline$\triangle \mathrm{CAD}$ & 1.457 & $8.676^{* *}$ & - \\
\hline \multirow[t]{2}{*}{ All } & $14.364 * * *$ & $621.348^{* * *}$ & $16.683 * * *$ \\
\hline & $\Delta \mathrm{GB} 3 \mathrm{Y}$ & $\Delta \mathrm{TB} 3 \mathrm{M}$ & $\Delta \mathrm{CAD}$ \\
\hline$\Delta \mathrm{GB} 3 \mathrm{Y}$ & - & $438.127 * * *$ & $16.021 * * *$ \\
\hline$\Delta \mathrm{TB} 3 \mathrm{M}$ & $6.873 * *$ & - & 3.459 \\
\hline$\triangle \mathrm{CAD}$ & 3.297 & $9.240 * * *$ & - \\
\hline \multirow{2}{*}{ All } & $9.797 * *$ & $447.704 * * *$ & $19.239 * * *$ \\
\hline & $\Delta \mathrm{GB} 5 \mathrm{Y}$ & $\Delta \mathrm{TB} 3 \mathrm{M}$ & $\Delta \mathrm{CAD}$ \\
\hline$\Delta \mathrm{GB} 5 \mathrm{Y}$ & - & $383.026^{* * *}$ & $21.687 * * *$ \\
\hline$\Delta \mathrm{TB} 3 \mathrm{M}$ & $9.130 * *$ & - & 3.583 \\
\hline$\triangle \mathrm{CAD}$ & 0.799 & $11.307 * * *$ & - \\
\hline \multirow[t]{2}{*}{ All } & $9.656 * *$ & $392.438 * * *$ & $24.913 * * *$ \\
\hline & $\Delta \mathrm{GB} 7 \mathrm{Y}$ & $\Delta \mathrm{TB} 3 \mathrm{M}$ & $\Delta \mathrm{CAD}$ \\
\hline$\Delta \mathrm{GB} 7 \mathrm{Y}$ & - & $281.376^{* * *}$ & $15.738 * * *$ \\
\hline$\Delta \mathrm{TB} 3 \mathrm{M}$ & $8.403 * *$ & - & 2.967 \\
\hline$\triangle \mathrm{CAD}$ & 0.341 & $12.696^{* * *}$ & - \\
\hline \multirow[t]{2}{*}{ All } & $8.880 *$ & $290.534 * * *$ & $18.961 * * *$ \\
\hline & $\Delta \mathrm{GB} 10 \mathrm{Y}$ & $\Delta \mathrm{TB} 3 \mathrm{M}$ & $\triangle \mathrm{CAD}$ \\
\hline$\Delta \mathrm{GB} 10 \mathrm{Y}$ & - & $233.051 * * *$ & $21.596 * * *$ \\
\hline$\Delta \mathrm{TB} 3 \mathrm{M}$ & 2.821 & - & 2.795 \\
\hline$\triangle \mathrm{CAD}$ & 0.422 & $11.011^{* * *}$ & - \\
\hline \multirow[t]{2}{*}{ All } & 3.156 & $242.100 * * *$ & $24.823 * * *$ \\
\hline & $\Delta \mathrm{GB} 30 \mathrm{Y}$ & $\Delta \mathrm{TB} 3 \mathrm{M}$ & $\Delta \mathrm{CAD}$ \\
\hline$\Delta \mathrm{GB} 30 \mathrm{Y}$ & - & $191.280 * * *$ & $7.221 * *$ \\
\hline$\triangle \mathrm{TB} 3 \mathrm{M}$ & 2.003 & - & 3.221 \\
\hline$\triangle \mathrm{CAD}$ & 0.165 & $10.107 * * *$ & - \\
\hline All & 2.253 & $198.577 * * *$ & $10.239 * *$ \\
\hline
\end{tabular}

Note: $* * *, * *$, and $*$ indicate statistical significance at the 1 percent, 5 percent, and 10 percent levels, respectively. 
Table 20: Granger Causality within the VECM Framework (equation 6)

\begin{tabular}{|c|c|c|c|}
\hline \multirow[b]{2}{*}{ Excluded } & \multicolumn{3}{|c|}{ Dependent Variable } \\
\hline & $\Delta \mathrm{GB} 2 \mathrm{Y}$ & $\Delta \mathrm{TB} 3 \mathrm{M}$ & $\Delta \mathrm{LN}[\mathrm{BRENT}]$ \\
\hline$\Delta \mathrm{GB} 2 \mathrm{Y}$ & - & $609.924 * * *$ & 2.981 \\
\hline$\Delta \mathrm{TB} 3 \mathrm{M}$ & $12.840 * * *$ & - & 0.667 \\
\hline$\Delta \mathrm{LN}[\mathrm{BRENT}]$ & 0.014 & 0.968 & - \\
\hline \multirow[t]{2}{*}{ All } & $12.849 * *$ & 611.012 & 4.663 \\
\hline & $\Delta \mathrm{GB} 3 \mathrm{Y}$ & $\Delta \mathrm{TB} 3 \mathrm{M}$ & $\Delta \mathrm{LN}[\mathrm{BRENT}]$ \\
\hline$\Delta \mathrm{GB} 3 \mathrm{Y}$ & - & $436.563 * * *$ & 2.929 \\
\hline$\Delta \mathrm{TB} 3 \mathrm{M}$ & $6.454 * *$ & - & 0.378 \\
\hline$\Delta \mathrm{LN}[\mathrm{BRENT}]$ & 0.787 & 0.761 & - \\
\hline \multirow{2}{*}{ All } & 7.225 & $437.766 * * *$ & 4.618 \\
\hline & $\Delta \mathrm{GB} 5 \mathrm{Y}$ & $\Delta \mathrm{TB} 3 \mathrm{M}$ & $\Delta \mathrm{LN}[\mathrm{BRENT}]$ \\
\hline$\Delta \mathrm{GB} 5 \mathrm{Y}$ & - & $379.441 * * *$ & 3.844 \\
\hline$\Delta \mathrm{TB} 3 \mathrm{M}$ & $8.825 * *$ & - & 0.761 \\
\hline$\Delta \mathrm{LN}[\mathrm{BRENT}]$ & 0.292 & 0.871 & - \\
\hline \multirow[t]{2}{*}{ All } & 9.113* & $380.776 * * *$ & 5.530 \\
\hline & $\Delta \mathrm{GB} 7 \mathrm{Y}$ & $\Delta \mathrm{TB} 3 \mathrm{M}$ & $\Delta \mathrm{LN}[\mathrm{BRENT}]$ \\
\hline$\Delta \mathrm{GB} 7 \mathrm{Y}$ & - & $276.406^{* * *}$ & $5.295^{*}$ \\
\hline$\Delta \mathrm{TB} 3 \mathrm{M}$ & $8.530 * *$ & - & 0.789 \\
\hline$\Delta \mathrm{LN}[\mathrm{BRENT}]$ & 0.326 & 0.894 & - \\
\hline \multirow[t]{2}{*}{ All } & $8.843^{*}$ & $277.832 * * *$ & 6.981 \\
\hline & $\Delta \mathrm{GB} 10 \mathrm{Y}$ & $\Delta \mathrm{TB} 3 \mathrm{M}$ & $\Delta \mathrm{LN}[\mathrm{BRENT}]$ \\
\hline$\Delta \mathrm{GB} 10 \mathrm{Y}$ & - & $230.212 * * *$ & $12.828 * * *$ \\
\hline$\Delta \mathrm{TB} 3 \mathrm{M}$ & 2.731 & - & 0.867 \\
\hline$\Delta \mathrm{LN}[\mathrm{BRENT}]$ & 0.563 & 1.050 & - \\
\hline \multirow[t]{2}{*}{ All } & 3.295 & $231.714 * * *$ & $14.513 * * *$ \\
\hline & $\Delta \mathrm{GB} 30 \mathrm{Y}$ & $\Delta \mathrm{TB} 3 \mathrm{M}$ & $\Delta \mathrm{LN}[\mathrm{BRENT}]$ \\
\hline$\Delta \mathrm{GB} 30 \mathrm{Y}$ & - & $188.701 * * *$ & $7.986^{* *}$ \\
\hline$\Delta \mathrm{TB} 3 \mathrm{M}$ & 2.033 & - & 1.167 \\
\hline$\Delta \mathrm{LN}[\mathrm{BRENT}]$ & 1.999 & 2.157 & - \\
\hline All & 4.072 & $190.931 * * *$ & $10.098 * *$ \\
\hline
\end{tabular}

Note: $* * *, * *$, and * indicate statistical significance at the 1 percent, 5 percent, and 10 percent levels, respectively. 
Table 21: Granger Causality within the VECM Framework (equation 7)

\begin{tabular}{|c|c|c|c|c|}
\hline \multirow[b]{2}{*}{ Excluded } & \multicolumn{4}{|c|}{ Dependent Variable } \\
\hline & $\Delta \mathrm{GB} 2 \mathrm{Y}$ & $\triangle \mathrm{TB} 3 \mathrm{M}$ & $\Delta \mathrm{LN}[\mathrm{TSX}]$ & $\Delta \mathrm{LN}[\mathrm{BRENT}]$ \\
\hline$\Delta \mathrm{GB} 2 \mathrm{Y}$ & - & $613.400 * * *$ & $14.931 * * *$ & 1.878 \\
\hline$\Delta \mathrm{TB} 3 \mathrm{M}$ & $12.630^{* * *}$ & - & $7.867 * *$ & 1.856 \\
\hline$\Delta \mathrm{LN}[\mathrm{TSX}]$ & $9.456 * * *$ & $12.901 * * *$ & - & $127.533 * * *$ \\
\hline$\Delta \mathrm{LN}[\mathrm{BRENT}]$ & 0.021 & 1.151 & 0.451 & - \\
\hline \multirow[t]{2}{*}{ All } & $22.366^{* * *}$ & $620.716^{* * *}$ & $33.256^{* * *}$ & 131.727 \\
\hline & $\Delta \mathrm{GB} 3 \mathrm{Y}$ & $\Delta \mathrm{TB} 3 \mathrm{M}$ & $\Delta \mathrm{LN}[\mathrm{TSX}]$ & $\Delta \mathrm{LN}[\mathrm{BRENT}]$ \\
\hline$\Delta \mathrm{GB} 3 \mathrm{Y}$ & - & $438.995 * * *$ & $14.884 * * *$ & 1.617 \\
\hline$\triangle \mathrm{TB} 3 \mathrm{M}$ & $6.180^{* *}$ & - & $9.571 * * *$ & 1.363 \\
\hline$\Delta \mathrm{LN}[\mathrm{TSX}]$ & $9.399 * * *$ & $11.929 * * *$ & - & $127.166^{* * *}$ \\
\hline$\Delta \mathrm{LN}[\mathrm{BRENT}]$ & 0.962 & 0.899 & 0.489 & - \\
\hline \multirow[t]{2}{*}{ All } & $16.696^{* *}$ & 446.314 & $33.246^{* * *}$ & $131.179 * * *$ \\
\hline & $\Delta \mathrm{GB} 5 \mathrm{Y}$ & $\Delta \mathrm{TB} 3 \mathrm{M}$ & $\Delta \mathrm{LN}[\mathrm{TSX}]$ & $\Delta \mathrm{LN}[\mathrm{BRENT}]$ \\
\hline$\Delta \mathrm{GB} 5 \mathrm{Y}$ & - & $383.982 * * *$ & $18.588^{* * *}$ & 2.189 \\
\hline$\Delta \mathrm{TB} 3 \mathrm{M}$ & $8.705 * *$ & - & $10.090 * * *$ & 2.200 \\
\hline$\Delta \mathrm{LN}[\mathrm{TSX}]$ & $12.622 * * *$ & $14.668 * * *$ & - & $126.065 * * *$ \\
\hline$\Delta \mathrm{LN}[\mathrm{BRENT}]$ & 0.339 & 0.882 & 0.548 & - \\
\hline \multirow[t]{2}{*}{ All } & $21.914 * * *$ & $391.631 * * *$ & $37.094 * * *$ & 131.031 \\
\hline & $\Delta \mathrm{GB} 7 \mathrm{Y}$ & $\Delta \mathrm{TB} 3 \mathrm{M}$ & $\Delta \mathrm{LN}[\mathrm{TSX}]$ & $\Delta \mathrm{LN}[\mathrm{BRENT}]$ \\
\hline$\Delta \mathrm{GB} 7 \mathrm{Y}$ & - & $279.292 * * *$ & $21.697 * * *$ & 3.327 \\
\hline$\Delta \mathrm{TB} 3 \mathrm{M}$ & $8.372 * *$ & - & $9.860 * * *$ & 2.184 \\
\hline$\Delta \mathrm{LN}[\mathrm{TSX}]$ & $14.660 * * *$ & $13.236^{* * *}$ & - & $125.466 * * *$ \\
\hline$\Delta \mathrm{LN}[\mathrm{BRENT}]$ & 0.402 & 0.885 & 0.632 & - \\
\hline \multirow{2}{*}{ All } & $23.697 * * *$ & $286.895 * * *$ & $40.180 * * *$ & 131.853 \\
\hline & $\Delta \mathrm{GB} 10 \mathrm{Y}$ & $\Delta \mathrm{TB} 3 \mathrm{M}$ & $\Delta \mathrm{LN}[\mathrm{TSX}]$ & $\Delta \mathrm{LN}[\mathrm{BRENT}]$ \\
\hline$\Delta \mathrm{GB} 10 \mathrm{Y}$ & - & $232.867 * * *$ & 23.325 & $9.772 * * *$ \\
\hline$\Delta \mathrm{TB} 3 \mathrm{M}$ & 2.593 & - & 10.741 & 2.192 \\
\hline$\Delta \mathrm{LN}[\mathrm{TSX}]$ & $11.659^{* * *}$ & $12.740 * * *$ & - & $124.047 * * *$ \\
\hline$\Delta \mathrm{LN}[\mathrm{BRENT}]$ & 0.681 & 1.002 & 0.701 & \\
\hline \multirow[t]{2}{*}{ All } & $15.095 * *$ & $240.536^{* * *}$ & $41 / 752 * * *$ & $137 / 837 * * *$ \\
\hline & $\Delta \mathrm{GB} 30 \mathrm{Y}$ & $\Delta \mathrm{TB} 3 \mathrm{M}$ & $\Delta \mathrm{LN}[\mathrm{TSX}]$ & $\Delta \mathrm{LN}[\mathrm{BRENT}]$ \\
\hline$\Delta \mathrm{GB} 30 \mathrm{Y}$ & - & $190.107 * * *$ & $14.547 * * *$ & $5.262^{*}$ \\
\hline$\Delta \mathrm{TB} 3 \mathrm{M}$ & 2.086 & - & $12.575^{* * *}$ & 2.801 \\
\hline$\Delta \mathrm{LN}[\mathrm{TSX}]$ & $4.988^{*}$ & $11.184 * * *$ & - & - \\
\hline$\Delta \mathrm{LN}[\mathrm{BRENT}]$ & 1.871 & 2.272 & 1.553 & $141.503 * * *$ \\
\hline All & 9.142 & $198.617 * * *$ & $33.502 * * *$ & $151.020 * * *$ \\
\hline
\end{tabular}

Note: $* * *, * *$, and $*$ indicate statistical significance at the 1 percent, 5 percent, and 10 percent levels, respectively. 
Table 22: Granger Causality within the VECM Framework (equation 8)

\begin{tabular}{|c|c|c|c|c|}
\hline \multirow[b]{2}{*}{ Excluded } & \multicolumn{4}{|c|}{ Dependent Variable } \\
\hline & $\Delta \mathrm{GB} 2 \mathrm{Y}$ & $\Delta \mathrm{TB} 3 \mathrm{M}$ & $\Delta \mathrm{LN}[\mathrm{TSX}]$ & $\triangle \mathrm{CAD}$ \\
\hline$\Delta \mathrm{GB} 2 \mathrm{Y}$ & - & $611.594 * * *$ & $14.316^{* * *}$ & $11.397 * * *$ \\
\hline$\triangle \mathrm{TB} 3 \mathrm{M}$ & $12.882 * * *$ & - & $7.398 * *$ & 3.076 \\
\hline$\Delta \mathrm{LN}[\mathrm{TSX}]$ & $9.001 * *$ & $8.985 * *$ & - & $179.009 * * *$ \\
\hline$\triangle \mathrm{CAD}$ & 1.024 & $5.273 *$ & $19.647 * * *$ & - \\
\hline \multirow[t]{2}{*}{ All } & $23.368 * * *$ & $624.978^{* * *}$ & $52.610^{* * *}$ & $195.773 * * *$ \\
\hline & $\Delta \mathrm{GB} 3 \mathrm{Y}$ & $\Delta \mathrm{TB} 3 \mathrm{M}$ & $\Delta \mathrm{LN}[\mathrm{TSX}]$ & $\triangle \mathrm{CAD}$ \\
\hline$\Delta \mathrm{GB} 3 \mathrm{Y}$ & - & $438.712 * * *$ & $14.301 * * *$ & $12.179 * * *$ \\
\hline$\Delta \mathrm{TB} 3 \mathrm{M}$ & $6.651 * *$ & - & $9.090 * *$ & 2.314 \\
\hline$\Delta \mathrm{LN}[\mathrm{TSX}]$ & $9.241 * * *$ & $7.979 * *$ & - & $177.142 * * *$ \\
\hline$\triangle \mathrm{CAD}$ & 3.352 & $5.895^{*}$ & $19.724 * * *$ & - \\
\hline \multirow[t]{2}{*}{ All } & $19.085 * * *$ & $451.439 * * *$ & $52.611 * * *$ & 196.608 \\
\hline & $\Delta \mathrm{GB} 5 \mathrm{Y}$ & $\Delta \mathrm{TB} 3 \mathrm{M}$ & $\Delta \mathrm{LN}[\mathrm{TSX}]$ & $\triangle \mathrm{CAD}$ \\
\hline$\Delta \mathrm{GB} 5 \mathrm{Y}$ & - & $385.474 * * *$ & $18.215 * * *$ & $15.364 * * *$ \\
\hline$\triangle \mathrm{TB} 3 \mathrm{M}$ & $9.029 * *$ & - & $9.557 * * *$ & 2.368 \\
\hline$\Delta \mathrm{LN}[\mathrm{TSX}]$ & $12.404 * * *$ & $9.873 * * *$ & - & $174.480 * * *$ \\
\hline$\triangle \mathrm{CAD}$ & 0.671 & $7.269 * *$ & $20.049 * * *$ & - \\
\hline \multirow[t]{2}{*}{ All } & $22.251 * * *$ & $398.219 * * *$ & $56.661 * * *$ & $199.694 * * *$ \\
\hline & $\Delta \mathrm{GB} 7 \mathrm{Y}$ & $\Delta \mathrm{TB} 3 \mathrm{M}$ & $\Delta \mathrm{LN}[\mathrm{TSX}]$ & $\Delta \mathrm{CAD}$ \\
\hline$\Delta \mathrm{GB} 7 \mathrm{Y}$ & - & $282.386^{* * *}$ & $22.203 * * *$ & $10.078 * * *$ \\
\hline$\triangle \mathrm{TB} 3 \mathrm{M}$ & $8.370 * *$ & - & $9.346 * * *$ & 1.754 \\
\hline$\Delta \mathrm{LN}[\mathrm{TSX}]$ & $14.281 * * *$ & $8.359 * *$ & - & $175.072 * * *$ \\
\hline$\triangle \mathrm{CAD}$ & 0.037 & $8.647 * *$ & $21.008 * * *$ & - \\
\hline \multirow[t]{2}{*}{ All } & $23.328 * * *$ & $294.871 * * *$ & $60.648 * * *$ & $194.318^{* * *}$ \\
\hline & $\Delta \mathrm{GB} 10 \mathrm{Y}$ & $\Delta \mathrm{TB} 3 \mathrm{M}$ & $\Delta \mathrm{LN}[\mathrm{TSX}]$ & $\Delta \mathrm{CAD}$ \\
\hline$\Delta \mathrm{GB} 10 \mathrm{Y}$ & - & $234.641 * * *$ & $23.421 * * *$ & $14.449 * * *$ \\
\hline$\triangle \mathrm{TB} 3 \mathrm{M}$ & 2.750 & - & $10.244 * * *$ & 1.644 \\
\hline$\Delta \mathrm{LN}[\mathrm{TSX}]$ & $11.555 * * *$ & $8.144 * *$ & - & $173.507^{* * *}$ \\
\hline$\triangle \mathrm{CAD}$ & 0.463 & $7.347 * *$ & $20.690 * * *$ & - \\
\hline \multirow[t]{2}{*}{ All } & $14.891 * *$ & $247.020 * * *$ & $61.786 * * *$ & $198.635 * * *$ \\
\hline & $\Delta$ GB30Y & $\Delta \mathrm{TB} 3 \mathrm{M}$ & $\Delta \mathrm{LN}[\mathrm{TSX}]$ & $\triangle \mathrm{CAD}$ \\
\hline$\Delta \mathrm{GB} 30 \mathrm{Y}$ & - & $192.317 * * *$ & $15.086 * * *$ & $6.659 * *$ \\
\hline$\Delta \mathrm{TB} 3 \mathrm{M}$ & 2.095 & - & $11.811 * * *$ & 1.766 \\
\hline$\Delta \mathrm{LN}[\mathrm{TSX}]$ & $4.973 *$ & $6.391 * *$ & - & $175.939 * * *$ \\
\hline$\triangle \mathrm{CAD}$ & 0.007 & $6.722 * *$ & $21.089 * * *$ & - \\
\hline All & 7.286 & $202.868 * * *$ & $52.975 * * *$ & $186.268^{* * *}$ \\
\hline
\end{tabular}

Note: $* * *, * *$, and $*$ indicate statistical significance at the 1 percent, 5 percent, and 10 percent levels, respectively. 
Table 23: Granger Causality within the VECM Framework (equation 9)

\begin{tabular}{|c|c|c|c|c|}
\hline \multirow[b]{2}{*}{ Excluded } & \multicolumn{4}{|c|}{ Dependent Variable } \\
\hline & $\Delta \mathrm{GB} 2 \mathrm{Y}$ & $\triangle \mathrm{TB} 3 \mathrm{M}$ & $\Delta \mathrm{LN}[\mathrm{BRENT}]$ & $\triangle \mathrm{CAD}$ \\
\hline$\Delta \mathrm{GB} 2 \mathrm{Y}$ & - & $609.291 * * *$ & 4.157 & $12.206 * * *$ \\
\hline$\Delta \mathrm{TB} 3 \mathrm{M}$ & $13.036^{* * *}$ & - & 2.631 & 4.401 \\
\hline$\Delta \mathrm{LN}[\mathrm{BRENT}]$ & 0.001 & 1.401 & - & $11.567 * * *$ \\
\hline$\triangle \mathrm{CAD}$ & 1.461 & $8.765 * *$ & $157.339 * * *$ & - \\
\hline \multirow[t]{2}{*}{ All } & $14.344 * *$ & $620.585 * * *$ & $162.445 * * *$ & $27.605 * * *$ \\
\hline & $\Delta \mathrm{GB} 3 \mathrm{Y}$ & $\Delta \mathrm{TB} 3 \mathrm{M}$ & $\Delta \mathrm{LN}[\mathrm{BRENT}]$ & $\triangle \mathrm{CAD}$ \\
\hline$\Delta \mathrm{GB} 3 \mathrm{Y}$ & - & $437.173 * * *$ & 2.908 & $14.672 * * *$ \\
\hline$\Delta \mathrm{TB} 3 \mathrm{M}$ & $6.853 * * *$ & - & 2.227 & 3.613 \\
\hline$\Delta \mathrm{LN}[\mathrm{BRENT}]$ & 0.681 & 1.135 & - & $11.369 * * *$ \\
\hline$\triangle \mathrm{CAD}$ & 3.194 & $9.255 * * *$ & $155.634 * * *$ & - \\
\hline \multirow[t]{2}{*}{ All } & 10.480 & $447.987 * * *$ & $160.816^{* * *}$ & $30.023 * * *$ \\
\hline & $\Delta \mathrm{GB} 5 \mathrm{Y}$ & $\Delta \mathrm{TB} 3 \mathrm{M}$ & $\Delta$ LN[BRENT] & $\Delta \mathrm{CAD}$ \\
\hline$\Delta \mathrm{GB} 5 \mathrm{Y}$ & - & $382.228 * * *$ & 4.328 & $19.794 * * *$ \\
\hline$\Delta \mathrm{TB} 3 \mathrm{M}$ & $9.205 * *$ & - & 3.292 & 3.771 \\
\hline$\Delta \mathrm{LN}[\mathrm{BRENT}]$ & 0.252 & 1.128 & - & $10.603 * * *$ \\
\hline$\triangle \mathrm{CAD}$ & 0.734 & $11.198^{* * *}$ & $155.767 * * *$ & - \\
\hline \multirow[t]{2}{*}{ All } & 10.005 & $393.091 * * *$ & $161.875 * * *$ & $34.949 * * *$ \\
\hline & $\Delta \mathrm{GB} 7 \mathrm{Y}$ & $\Delta \mathrm{TB} 3 \mathrm{M}$ & $\Delta$ LN[BRENT] & $\triangle \mathrm{CAD}$ \\
\hline$\Delta \mathrm{GB} 7 \mathrm{Y}$ & - & $280.647 * * *$ & $5.006^{*}$ & $14.125 * * *$ \\
\hline$\Delta \mathrm{TB} 3 \mathrm{M}$ & $8.485^{* *}$ & - & 3.330 & 3.154 \\
\hline$\Delta \mathrm{LN}[\mathrm{BRENT}]$ & 0.357 & 1.172 & - & $10.567 * * *$ \\
\hline$\triangle \mathrm{CAD}$ & 0.386 & $12.678 * * *$ & $154.158 * * *$ & - \\
\hline \multirow[t]{2}{*}{ All } & 9.383 & $291.454 * * *$ & $161.786^{* * *}$ & $29.152 * * *$ \\
\hline & $\Delta \mathrm{GB} 10 \mathrm{Y}$ & $\Delta \mathrm{TB} 3 \mathrm{M}$ & $\Delta$ LN[BRENT] & $\Delta \mathrm{CAD}$ \\
\hline$\Delta \mathrm{GB} 10 \mathrm{Y}$ & - & $232.352 * * *$ & $13.203 * * *$ & $19.419 * * *$ \\
\hline$\Delta \mathrm{TB} 3 \mathrm{M}$ & 2.876 & - & 3.191 & 2.957 \\
\hline$\Delta \mathrm{LN}[\mathrm{BRENT}]$ & 0.550 & 1.308 & - & $9.932 * * *$ \\
\hline$\triangle \mathrm{CAD}$ & 0.389 & $11.095 * * *$ & $154.306^{* * *}$ & - \\
\hline \multirow[t]{2}{*}{ All } & 3.782 & $243.231 * * *$ & $169.743 * * *$ & $34.353 * * *$ \\
\hline & $\Delta \mathrm{GB} 30 \mathrm{Y}$ & $\Delta \mathrm{TB} 3 \mathrm{M}$ & $\Delta$ LN[BRENT] & $\Delta \mathrm{CAD}$ \\
\hline$\Delta \mathrm{GB} 30 \mathrm{Y}$ & - & $192.172 * * *$ & $6.255^{* *}$ & $6.067 * *$ \\
\hline$\Delta \mathrm{TB} 3 \mathrm{M}$ & 1.996 & - & 3.819 & 3.386 \\
\hline$\Delta \mathrm{LN}[\mathrm{BRENT}]$ & 1.898 & 3.169 & - & $13.277 * * *$ \\
\hline$\triangle \mathrm{CAD}$ & 0.163 & $11.504 * * *$ & $169.076^{* * *}$ & - \\
\hline All & 4.170 & $203.572 * * *$ & $179.438 * * *$ & $23.568 * * *$ \\
\hline
\end{tabular}

Note: $* * *, * *$, and $*$ indicate statistical significance at the 1 percent, 5 percent, and 10 percent levels, respectively.

\section{CONCLUSION}

This paper examines the Keynesian perspective on the relationship between Canadian government securities yields and the short-term interest rate by examining their long-run and short-run dynamics. A set of macroeconomic and financial variables are included in the regressions to control for the variables' potential impacts on government securities yields. There is ample evidence that the short-term and long-term interest rates are cointegrated in a wide range of models. 
The empirical findings reported in the paper have implications for both economic theory and public policy, as well as for the ongoing debate in macroeconomics as reflected in the discussions pertaining to the implementation of monetary policy and central bank operations (Bindseil 2004; Fullwiler 2008 [2017]; Kregel 2014; Lavoie 2014), the fiscal theory of price (Sims 2013), and other issues on monetary policy (Davidson 2015; Wray 2012).

The results obtained show evidence of short-run and long-run dynamics between Canadian government bond yields, the short-term interest rate, and other macroeconomic and financial variables, such as the exchange rate of the Canadian dollar, the log of the price of crude oil, and the log of the equity index. Therefore, one can argue that the Bank of Canada's actions affect Canadian government bond yields primarily through the short-term interest rate on Treasury bills. This supports Keynes's view that the central bank's actions influence the long-term interest rate on government bonds mainly through the effects of its actions on the short-term interest rate. The findings also show that while the short-term interest rate is an important driver of the longterm interest rate on Canadian government securities yields, other factors - such as the exchange rate of the Canadian dollar, the price of crude oil, and the equity index-also matter.

These findings are relevant for policy issues in Canada and elsewhere. The findings can inform the Bank of Canada in formulating its monetary policy, it can provide useful information to the monetary authorities in assessing and evaluating the monetary transmission, and it can also be useful for fiscal policy. The findings can inform private investors' portfolio decisions and views, as well as the Treasury's management of government debt. It also can help policymakers in making decisions concerning fiscal policy, and in assessing the impact of fiscal stimulus and contraction on long-term interest rates on Canadian government bonds. It can be of particular interest if these findings are supplemented with additional results obtained from macroeconomic models that incorporate quarterly macroeconomic data, especially concerning ratios of government debt and fiscal deficit as a share of national income. 


\section{REFERENCES}

Akram, T. 2014. “The Economics of Japan's Stagnation.” Business Economics 49(3): 156-75.

Akram, T., and A. Das. 2014. "Understanding the Low Yields of the Long-Term Japanese Sovereign Debt.” Journal of Economic Issues 48(2): 331-40.

— 2015. "A Keynesian Explanation of Indian Government Bond Yields." Journal of Post Keynesian Economics 38(4): 565-87.

_ 2017. "The Dynamics of Government Bond Yields in the Eurozone." Annals of Financial Economics 12(3): 1750011-1-1750011-18.

_ 2019a. "The Long-Run Determinants of Indian Government Bond Yields." Asian Development Review 36(1): 168-205.

—. 2019b. "An Analysis of the Daily Changes in US Treasury Security Yields." Levy Institute Working Papers No. 934. Annandale-on-Hudson, NY: Levy Economics Institute of Bard College.

—. Forthcoming. "Australian Government Bonds Nominal Yields: A Keynesian Perspective." Annals of Financial Economics.

Akram, T., and H. Li. 2016. “The Empirics of Long-Term US Interest Rates.” Levy Economics Working Papers No. 863. Annandale-on-Hudson, NY: Levy Economics Institute of Bard College.

— 2017. "What Keeps Long-Term U.S. Interest Rates So Low?" Economic Modelling 60: $380-90$.

—. 2018. “The Dynamics of JGBs Nominal Yields.” Levy Institute Working Paper No. 906. Annandale-on-Hudson, NY: Levy Economics Institute of Bard College.

_ 2019a. "The Impact of the Bank of Japan's Monetary Policy on Japanese Government Bonds' Low Nominal Yields.” Levy Economics Institute Working Paper No. 938. Annandale-on-Hudson, NY: Levy Economics Institute of Bard College.

_ 2019b. "An Inquiry Concerning Long-Term U.S. Interest Rates Using Monthly Data." Applied Economics. Available at: https://doi.org/10.1080/00036846.2019.1693696 
Ardagna, S., F. Caselli, and T. Lane. 2007. "Fiscal Discipline and the Cost of Public Debt Service: Some Estimates for OECD Countries.” The B. E. Journal of Macroeconomics 7(1): 1-33.

Baldacci, E., and M. Kumar. 2010. "Fiscal Deficits, Public Debt, and Sovereign Bond Yields." IMF Working Paper No. 10/184. Washington, DC: International Monetary Fund.

Bindseil, U. 2004. Monetary Policy Implementation: Theory, Past and Present. Oxford and New York: Oxford University Press.

Bollerslev, T., J. Cai, and F. M. Song. 2000. "Intraday Periodicity, Long Memory Volatility, and Macroeconomic Announcement Effects in the US Treasury Bond Market." Journal of Empirical Finance 7(1): 37-55.

Davidson, P. 2015. Post Keynesian Theory and Policy: A Realistic Analysis of the MarketOriented Capitalist Economy. Cheltenham, UK and Northampton, MA: Edward Elgar.

Dickey, D. A., and W. A. Fuller. 1979. "Distribution of the Estimators for Autoregressive Time Series with a Unit Root." Journal of the American Statistical Association 74(366): 42731.

1981. "Likelihood Ratio Statistics for Autoregressive Time Series with a Unit Root." Econometrica 49(4): 1057-72.

Doi, T., T. Hoshi, and T. Okimoto. 2011. "Japanese Government Debt and Sustainability of Fiscal Policy." Journal of the Japanese and International Economies 25(4): 414-33.

Elmendorf, D. W., and N. G. Mankiw. 1998. "Government Debt.” NBER Working Paper No. 6470. Cambridge, MA: National Bureau of Economic Research.

Fullwiler, S. T. 2008 [2017]. "Modern Central Bank Operations: The General Principles.” In Louis-Philippe Rochon and Sergio Rossi (eds.), Advances in Endogenous Money Analysis. Northampton, MA: Edward Elgar.

Gruber, J. W., and S. B. Kamin. 2012. "Fiscal Positions and Government Bond Yields in OECD Countries." Journal of Money, Credit, and Banking 44(8): 1563-87.

Gürkaynak, R. S., B. Sack, and J. H. Wright. 2007. "The US Treasury Yield Curve: 1961 to the Present." Journal of Monetary Economics 54(8): 2291-304. 
Hansen, G., and S. İmrohoroğlu. 2013. "Fiscal Reform and Government Debt in Japan: A Neoclassical Perspective." Review of Economic Dynamics 21: 201-24.

Horioka, C. Y., T. Nomoto, and A. Terada-Hagiwara. 2014. "Why Has Japan's Massive Government Debt Not Wreaked Havoc (Yet)?” The Japanese Political Economy 40(2): $3-23$.

Hoshi, T., and T. Ito. 2013. "Is the Sky the Limit? Can Japanese Government Bonds Continue to Defy Gravity?" Asian Economic Policy Review 8(2): 218-47.

—. 2014. "Defying Gravity: Can Japanese Sovereign Debt Continue to Increase Without a Crisis?” Economic Policy 29(77): 5-44.

Johansen, S. 1991. "Estimation and Hypothesis Testing of Cointegration Vectors in Gaussian Vector Autoregressive Models." Econometrica 59(6): 1551-80.

-1995. Likelihood-Based Inference in Cointegrated Vector Autoregressive Models. Oxford: Oxford University Press.

Keynes, J. M. 1930. A Treatise on Money, Vol. II: The Applied Theory of Money. London: Macmillan.

- [1936] 2007. The General Theory of Employment, Interest, and Money. New York: Palgrave Macmillan.

Kregel, J. 2011. "Was Keynes’ Monetary Policy À Outrance in the Treatise, A Forerunner of ZIRP and QE? Did He Change His Mind in the General Theory?" Levy Institute Policy Note No. 2011/4. Annandale-on-Hudson, NY: Levy Economics Institute of Bard College.

Lam, R. W., and K. Tokuoka. 2011. “Assessing the Risks to the Japanese Government Bond (JGB) Market." Journal of International Commerce, Economics and Policy 4(1): 1350002-1-1350002-15.

Lavoie, M. 2014. Post-Keynesian Economics: New Foundations. Cheltenham, UK and Northampton, MA: Edward Elgar.

Lerner, A. P. 1947. "Money as a Creature of the State.” American Economic Review 37(2): $312-$ 17. 
Levrero, E. S., and M. Deleidi. 2019. "The Causal Relationship Between Short- and Long-Term Interest Rates: An Empirical Assessment of the United States." MPRA Paper No. 93608. Available at: https://mpra.ub.uni-muenchen.de/93608/

Macrobond. Various years. Macrobond subscription services (accessed October 20, 2019).

Paccagnini, A. 2016. "The Macroeconomic Determinants of the US Term Structure during the Great Moderation.” Economic Modelling 52(A): 216-25.

Phillips, P. C. B., and P. Perron. 1988. "Testing for a Unit Root in Time Series Regression." Biometrika 75(2): 335-46.

Poghosyan, T. 2014. "Long-Run and Short-Run Determinants of Sovereign Bond Yields in Advanced Economies.” Economic Systems 38(1): 100-14.

Reinhart, C. M., and K. S. Rogoff. 2009. This Time Is Different: Eight Centuries of Financial Folly. Princeton, NJ: Princeton University Press.

Riefler, W. W. 1930. Money Rates and Money Markets in the United States. New York and London: Harper \& Brothers.

Simoski, S. 2019. "A Keynesian Exploration of the Determinants of Government Bond Yields for Brazil, Colombia, and Mexico." Master of Science thesis, Levy Economics Institute of Bard College. Annandale-on-Hudson, NY: Levy Economics Institute of Bard College.

Sims, C. A. 2013. "Paper Money.” American Economic Review 103(2): 563-84.

Tkačevs, O., and K. Vilerts. 2019. "The Impact of Government Borrowing Costs on Fiscal Discipline.” Kyklos 729(3): 446-71.

Tokuoka, K. 2012. “Intergenerational Implications of Fiscal Consolidation in Japan.” IMF Working Paper No. 12/197. Washington, DC: International Monetary Fund.

Wray, L. R. 2012. Modern Money Theory: A Primer on Macroeconomics for Sovereign Monetary Systems. New York: Palgrave Macmillan. 